\title{
A systematic review of gambling-related findings from the National Epidemiologic Survey on Alcohol and Related Conditions
}

\author{
JASMINE M. Y. LOO ${ }^{1,2}$, SHANE W. KRAUS ${ }^{3}$ and MARC N. POTENZA ${ }^{4,5,6,7 *}$ \\ ${ }^{1}$ School of Medicine and Health Sciences, Monash University Malaysia, Selangor Darul Ehsan, Malaysia \\ ${ }^{2}$ Research Department, The Salvation Army - Sydney Headquarters, Redfern, Sydney, NSW, Australia \\ ${ }^{3}$ Department of Psychology, University of Nevada, Las Vegas, NV, USA \\ ${ }^{4}$ Department of Psychiatry, Yale University School of Medicine, New Haven, CT, USA \\ ${ }^{5}$ Department of Neuroscience, Yale University School of Medicine, New Haven, CT, USA \\ ${ }^{6}$ Yale Child Study Center, Yale University School of Medicine, New Haven, CT, USA \\ ${ }^{7}$ The Connecticut Mental Health Center, New Haven, CT, USA
}

(Received: April 27, 2018; revised manuscript received: October 29, 2019; accepted: November 3, 2019)

\begin{abstract}
Background and aims: This systematic review analyzes and summarizes gambling-related findings from the nationally representative US National Epidemiological Survey on Alcohol and Related Conditions (NESARC) data. Methods: Systematic literature searches in accordance with PRISMA guidelines found 51 eligible studies that met inclusion criteria. Eight studies utilized both Waves 1 and 2 NESARC data, and selection of sample sizes varied from 185 to 43,093 individuals, consistent with specified research objectives of each study. Results: The prevalence of lifetime pathological gambling was $0.42 \%$ ( $0.64 \%$ among men, $0.23 \%$ among women), while past-year prevalence was $0.16 \%$. Pathological gambling rates were generally higher in populations with substance-use disorders and other psychiatric diagnoses. Rates of adverse childhood experiences and suicidal attempts were higher among individuals with problem or pathological gambling. Early-onset gamblers were more likely to be male, be never married, have incomes below $\$ 70,000$, belong to younger cohorts and have Cluster B personality disorders, but less likely to be diagnosed with mood disorders. While pathological gambling was related to obesity, increased stress, and poorer physical health among general age groups, recreational gambling was linked with improved physical and mental functioning in older adults. Conclusions: The NESARC has provided important information on the correlates of pathological gambling and subdiagnostic patterns of gambling behaviors. Additional studies should examine these relationships in the current gambling environment and longitudinally with aims of implementing policies to improve the public health.
\end{abstract}

Keywords: systematic review, NESARC, gambling, pathological gambling, national data sets

\section{INTRODUCTION}

Gambling may be defined as placing monetary or material items at risk in hopes of gaining money or items of greater material value. Gambling may be viewed along a severity continuum with "Pathological Gambling" in DSM-IV and ICD-10 (World Health Organization, 1992) and "Gambling Disorder" in DSM-5 referring to gambling behavior that leads to significant distress and interference with functioning in major life domains (American Psychiatric Association, 2013). Meanwhile, "Problem Gambling" refers to gambling that negatively affects functioning and relationships (Morasco et al., 2006) but may not meet DSM or ICD criteria for pathological gambling/gambling disorder; that is, the term problem gambling is often used to describe subdiagnostic levels of gambling that are concerning. Recreational gambling has been operationalized in the National Epidemiologic Survey on Alcohol and Related Conditions (NESARC) as lifetime gambling of more than five times per year without meeting more than two DSM criteria (Desai, Desai, \& Potenza, 2007). Other designations such as low-risk and at-risk gambling have been used and defined in the context of NESARC data, with designations relating to numbers of inclusionary criteria for pathological gambling acknowledged (Desai \& Potenza, 2008). These definitions will guide our discussion in this systematic review on gambling-related NESARC findings.

Nationally representative data provide important descriptive and inferential findings that assist in policy-making, industry compliance assessments, community development, and generation of improved treatment and prevention strategies. For example, data collected from the US National Comorbidity Survey Replication (NCS-R), a nationally

* Corresponding author: Marc N. Potenza, PhD, MD; Department of Psychiatry, Yale University School of Medicine, CMHC Room S-104, 34 Park St, New Haven, CT 06519, USA; Phone: +1 203 737 3553; Fax: +1 203737 3591; E-mail: marc.potenza@yale.edu

This is an open-access article distributed under the terms of the Creative Commons Attribution-NonCommercial 4.0 International License, which permits unrestricted use, distribution, and reproduction in any medium for non-commercial purposes, provided the original author and source are credited, a link to the CC License is provided, and changes - if any - are indicated. 
representative US household survey, assessed 9,282 English-speaking respondents aged 18 years and older and was conducted between February 2001 and April 2003 in a nationally representative multistage clustered area probability sample of the US household population. Specifically, the NCS-R found lifetime prevalence estimates of problem gambling (defined as having one or more criteria of pathological gambling) at $2.3 \%$ and pathological gambling at $0.6 \%$ (Kessler et al., 2008). In other countries, the British Gambling Prevalence Survey in 2010 - as a follow-up to the 1999 and 2007 surveys - was conducted to allow comparisons pre- and post-implementation of the Gambling Act 2005 (Wardle et al., 2011). Prior to this survey, the 2007 British Gambling Prevalence Survey aimed to examine the nature and extent of gambling in Great Britain before administering the Act. Similarly in Asia, the Singapore National Council on Problem Gambling has conducted gambling participation surveys every 3 years from 2005 to 2014 to guide development of systemic regulatory assessment, community engagement activities, prevention programs, and treatment provision (National Council on Problem Gambling, 2014). It is important that national follow-up surveys attempt to achieve maximal comparability with previous surveys by standardizing methodology and measurement instruments.

Comparing prevalence estimates between countries is often challenging as data-collection protocols, sampling procedures, and gambling assessments are often different, all of which may influence pathological gambling and problem gambling estimates. As such, prevalence estimates in this context may best be considered as an illustration of the range of worldwide approximations, and arguably not as definitive comparisons. Overall, national pathological gambling prevalence estimates in Western countries range from $0.3 \%$ in Sweden (Binde, 2014), 0.7\% in Britain (Wardle et al., 2011) to $0.8 \%$ in Denmark (Ekholm et al., 2014). In Australia and New Zealand, estimates range from $0.5 \%$ in New Zealand (Devlin \& Walton, 2012) to 2.1\% in Australia (Productivity Commission, 2010). Meanwhile, pathological gambling prevalence estimates in Asia are generally higher with a range from $0.5 \%$ in Singapore (National Council on Problem Gambling, 2014), 2.5\% in Macau (Fong \& Ozorio, 2005; Wu, Lai, \& Tong, 2014), 4\% in Hong Kong (Wong \& So, 2003) to $4.4 \%$ in Malaysia (Loo \& Ang, 2013). The prevalence rate observed in the US is like other Western countries, notably Sweden.

The National Institute on Alcohol Abuse and Alcoholism's NESARC is the largest ( $N=43,093$ adults) comorbidity survey conducted in the US that assessed DSM-IV pathological gambling and multiple substance-use, mood, anxiety, and personality disorders (Petry, Stinson, \& Grant, 2005). The nationally representative sample with statistical weights has enabled multivariate investigations of prevalence estimates, gender-related associations, sociodemographic correlates, potential risk factors, and physical and psychiatric comorbidities. Furthermore, NESARC data collected from Wave 1 (2001-2002) and Wave 2 (2004-2005) provide for a unique opportunity to evaluate longitudinal and prospective research questions, although pathological gambling was only assessed in Wave 1. To date, although 51 articles have reported pathological-gambling-related findings based on NESARC data, there have been no systematic reviews synthesizing results from more than a decade of publications. Such a summary could shed light on lessons learned from NESARC as well as inform future research efforts aimed at understanding the correlates and impact of pathological gambling and subdiagnostic levels.

A review of the gambling-related investigations from the NESARC data will help synthesize information gleaned from this data set and lay the foundation for future investigations of these and other data. This systematic review seeks to collect, evaluate, and discuss gambling-related NESARC findings, as the NESARC-related findings are important in informing policy makers, governmental bodies, researchers, and treatment providers. In the following sections, we will provide details on the systematic review methodology, evaluate findings from selected studies, and discuss implications for future directions. These findings are described and partitioned into relevant sections such as psychometrics, model testing, symptom analysis, prevalence estimates, gambling subtypes, sociodemographic correlates, potential risk factors, and comorbidities. Finally, "Discussion" section will highlight key findings, research and practical implications, and future directions.

\section{METHODS}

\section{Search strategy}

Protocols and strategies employed in this systematic review were in accordance with the Preferred Reporting Items for Systematic Reviews and Meta-Analyses (PRISMA) guidelines (Moher, Liberati, Tetzlaff, Altman, \& PRISMA Group, 2009). Electronic Boolean searches of databases (PsycInfo, PubMed, and Web of Science) included all articles from inception to June 2019. The search strategy included a combination of keywords such as "National Epidemiologic Survey on Alcohol and Related Conditions" or "NESARC" and "gambling," "pathological gambling," "problem gambling," or "gambling disorder." Only articles in English were selected in the three search engines. Reference lists of included articles were checked manually for additional relevant publications.

\section{Inclusion criteria and review process}

All citations were managed with Endnote reference management software. Each reference title and abstract was screened by the authors independently and discussed as a group for eligibility against the inclusion criteria: (a) original empirical publications in English, (b) studies included NESARC Wave 1 and/or Wave 2 data, and (c) studies reported data from the gambling component of the Alcohol Use Disorder and Associated Disabilities Interview Schedule (AUDADIS-IV). The resulting full-text copies of all articles considered to be relevant were retrieved and screened.

Data from included studies were extracted into MS Excel 2013 by the first author and independently checked by the other authors. Although the authors agreed upon the manuscripts to be included in the final analyses, data on independent evaluation for inclusion were not systematically 
collected. The following characteristics of each study were retrieved: (a) general information - title, authors, and publication year; (b) sample size and other characteristics; (c) instrument and thresholding for pathological gambling; (d) other variables investigated in each study; (e) statistical analyses; (f) main findings or prevalence of pathological gambling; and $(\mathrm{g})$ other findings.

\section{RESULTS}

\section{Study selection}

Upon removal of duplicates, the searches identified 67 records that were screened, whereby 51 empirical papers met the inclusion criteria and were included in this systematic review (see Figure 1 for PRISMA flow diagram). Nine excluded articles were not written based on NESARC data, while one excluded record was a conference abstract that was eventually published as an article and included in this systematic review. Details extracted from articles $(n=43)$ that examined pathological gambling as one of the main variables within specified research questions are summarized in Table 1. Information extracted from the remaining 8 articles (out of the 51 included) that investigated pathological gambling as a peripheral variable in the context of other psychopathology is summarized in Table 2.

\section{Study characteristics}

All 51 included studies utilized either Wave 1 (Year 20012002) and/or Wave 2 (2005-2006) nationally representative NESARC data (Grant, Moore, Shepard, \& Kaplan, 2003) and were published between years 2005 and 2019. Ten studies utilized both Wave 1 and 2 data sets for prospective evaluations of associations between pathological gambling at Wave 1 and medical/psychiatric disorders at Wave 2 . Selection of sample sizes varied from 185 to 43,093 individuals depending on specified sample selection built upon research objectives.

\section{Instrument}

Assessment of pathological-gambling criteria based on the Diagnostic and Statistical Manual of Mental Disorders, 4th edition (DSM-IV; American Psychiatric Association, 2000)
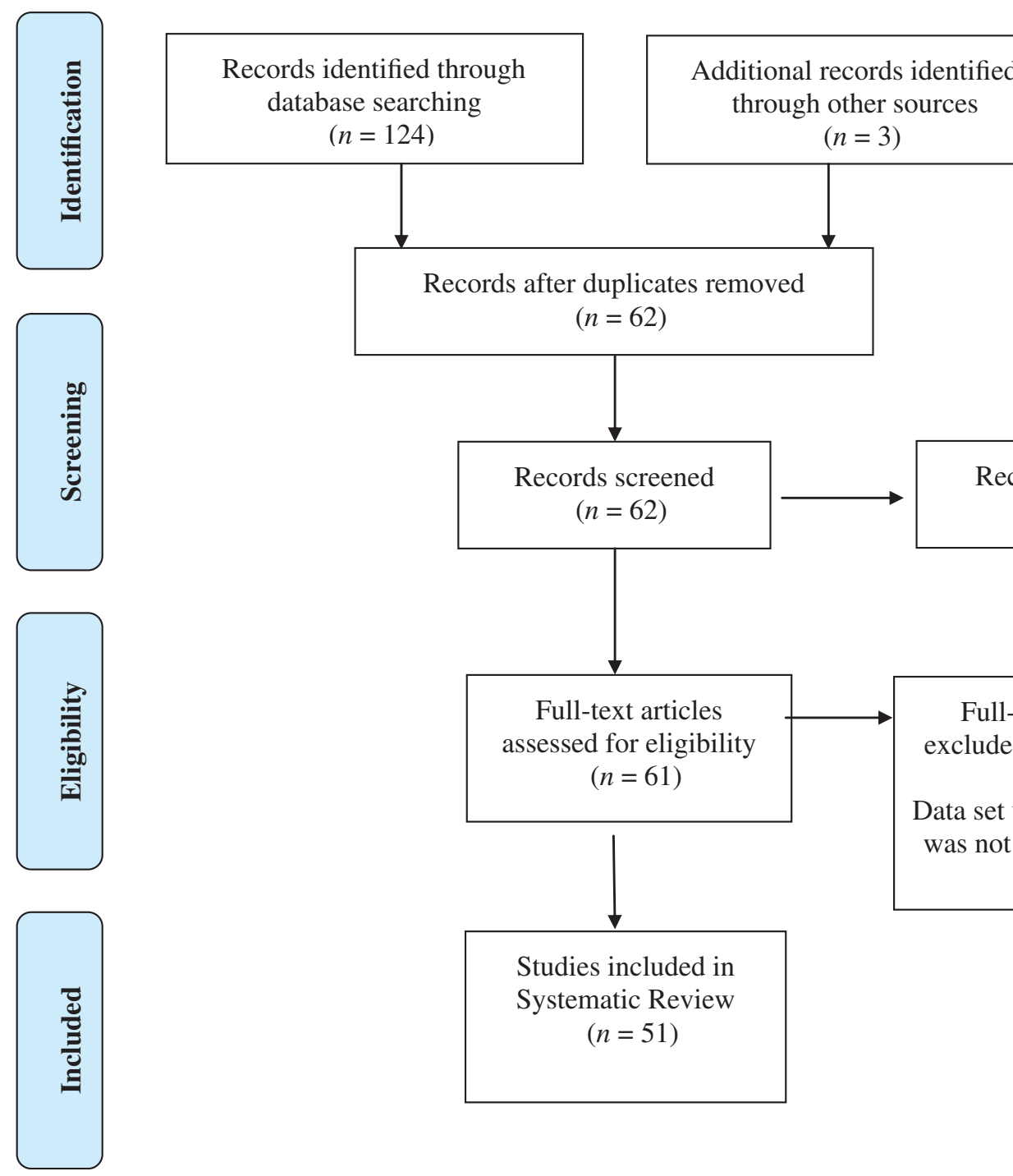
through other sources

$$
(n=124)
$$

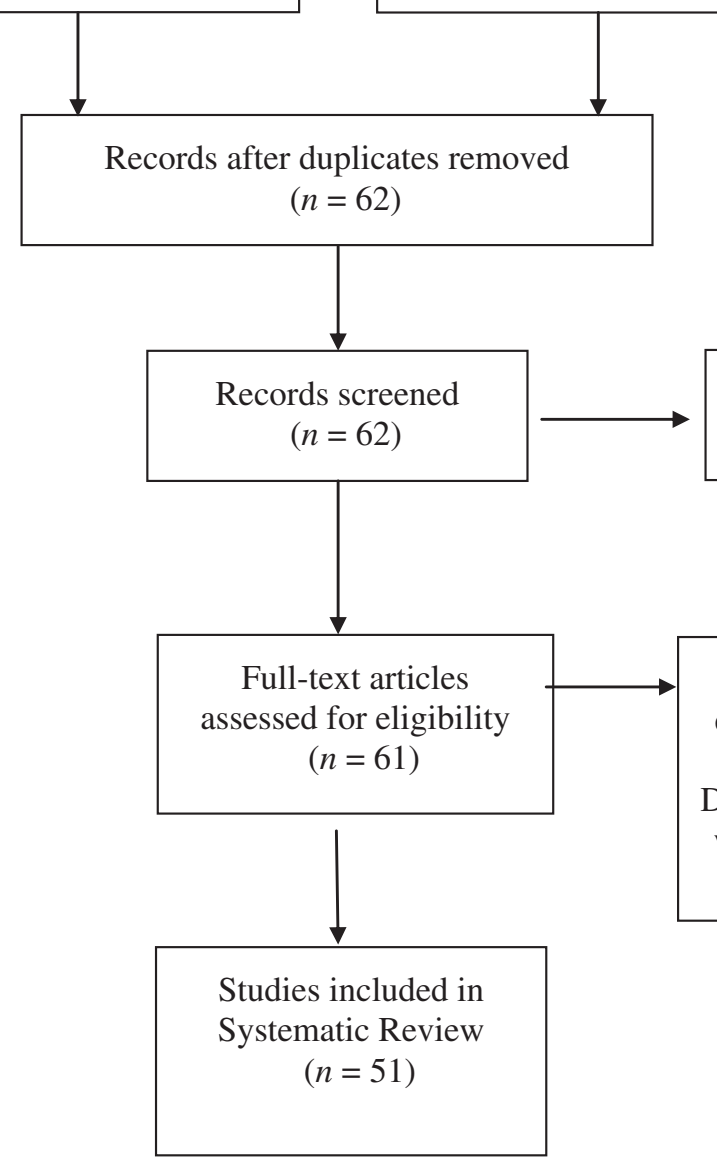

Records excluded $(n=1)$

Full-text articles excluded, with reasons $(n=10)$

Data set used for analysis was not NESARC data

Figure 1. PRISMA flow diagram of the systematic review phases 


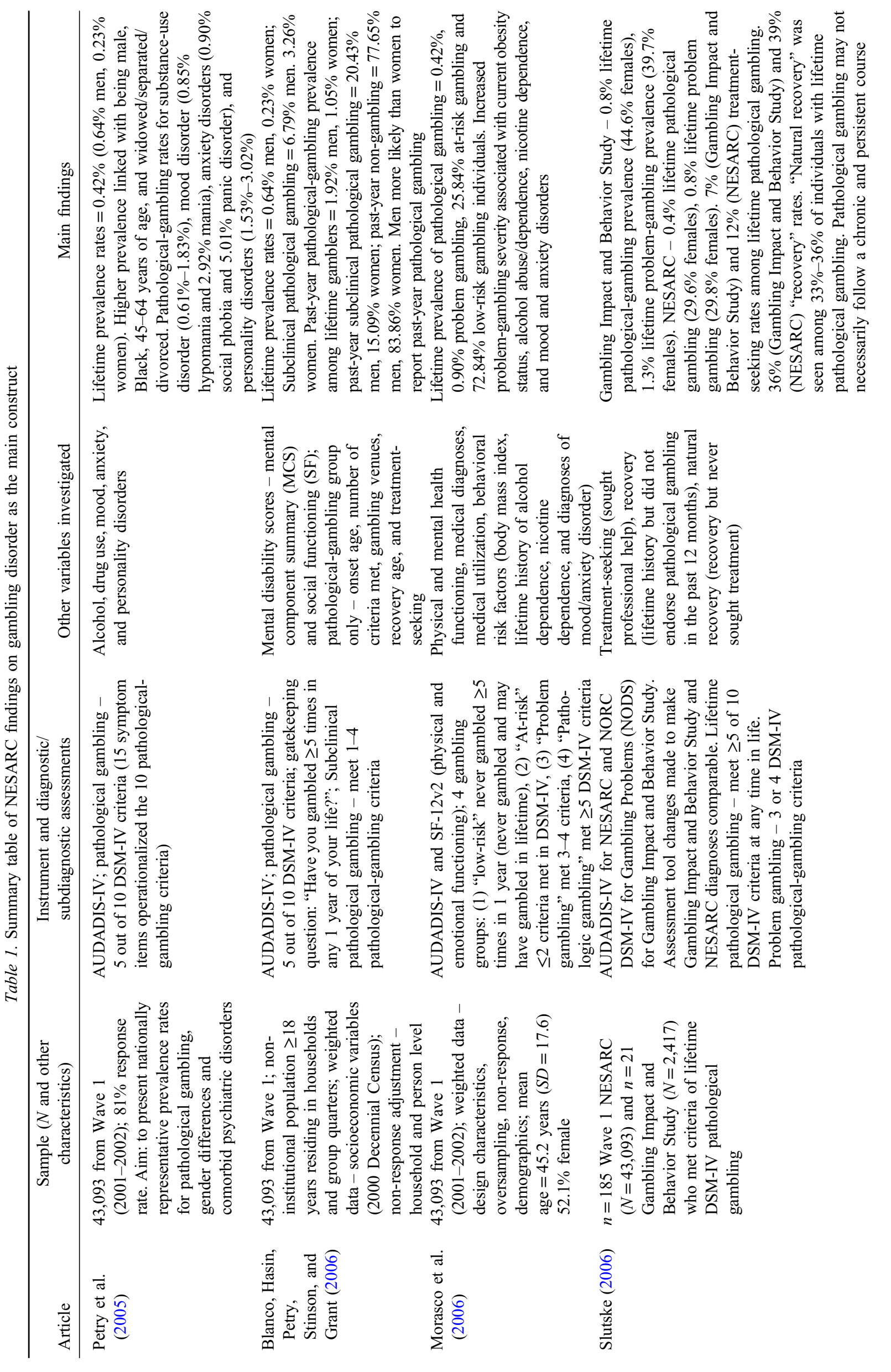




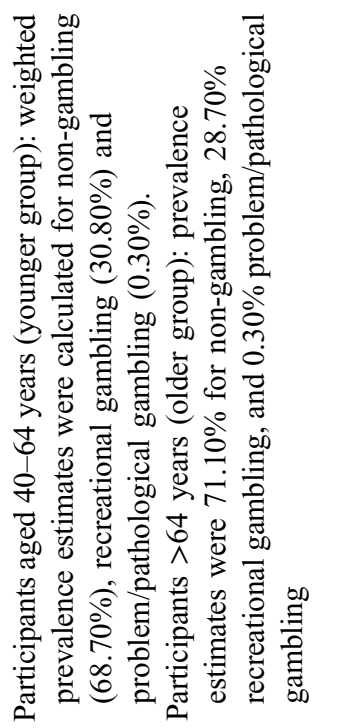

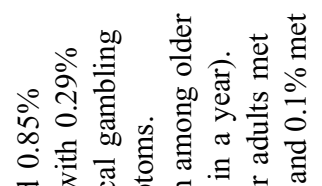

类

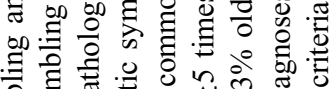

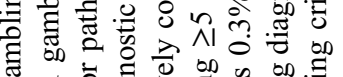

要

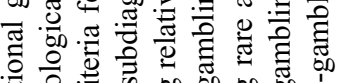

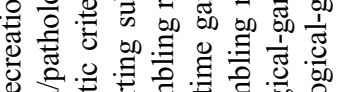

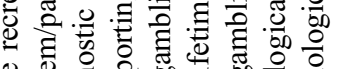

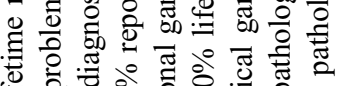

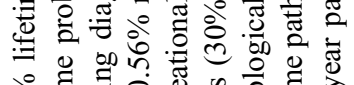

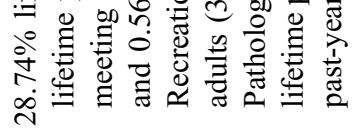

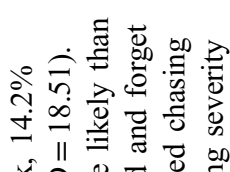

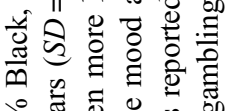

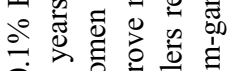

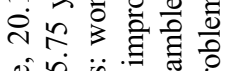

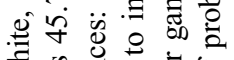

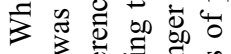

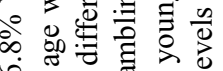
잉

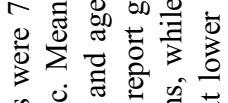
n.

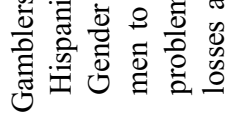

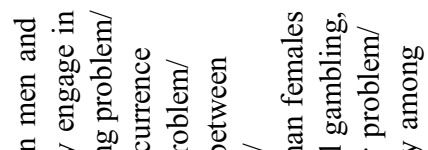

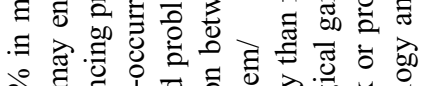

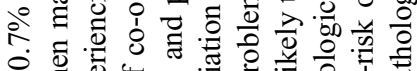
$\circ$ 过 4 o क्षे

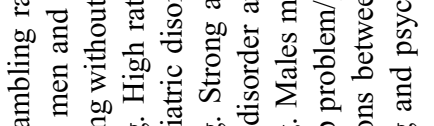

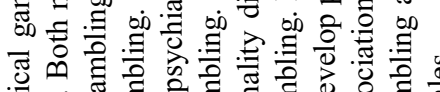

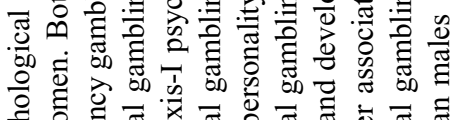

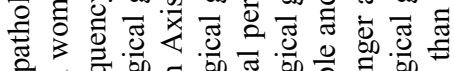
:

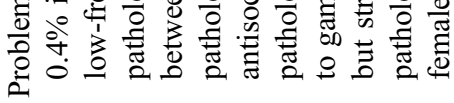

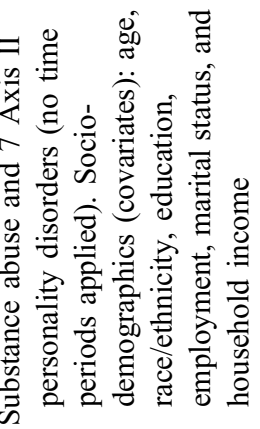

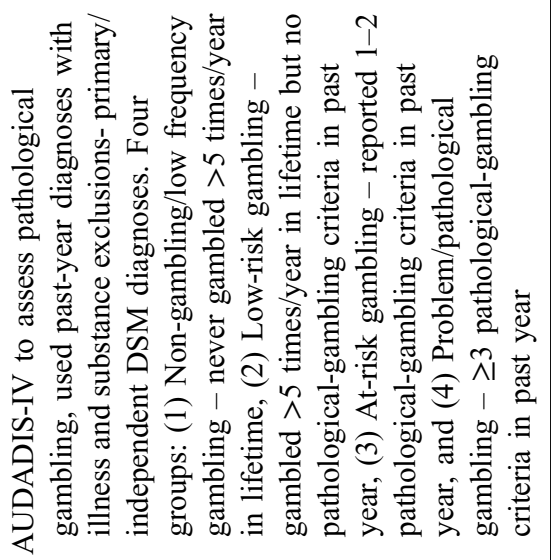

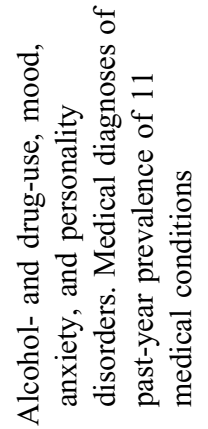

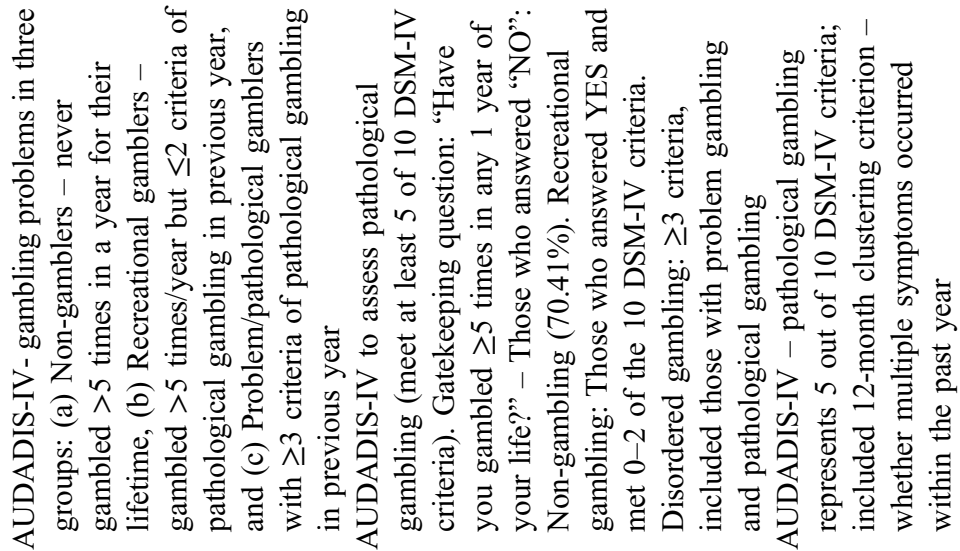

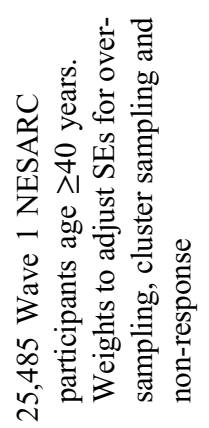

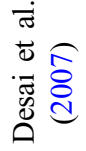

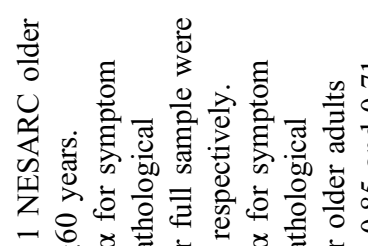

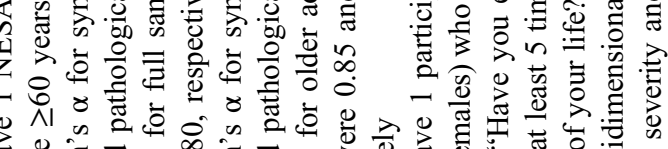

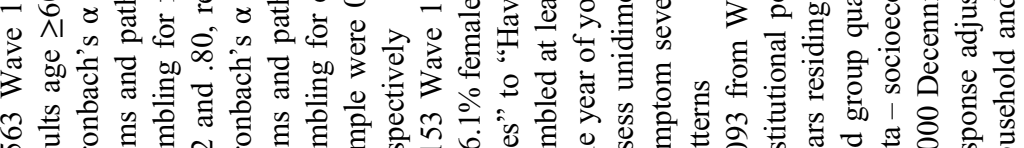

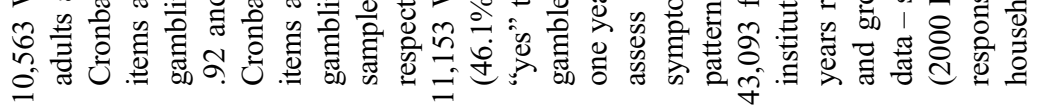

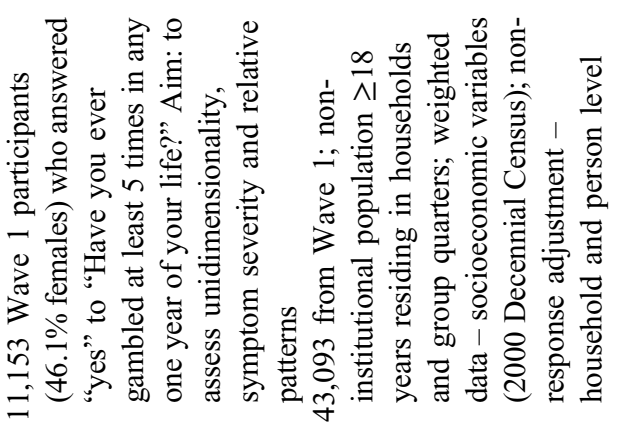

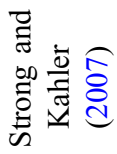

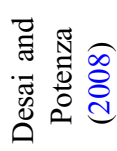


Loo et al.

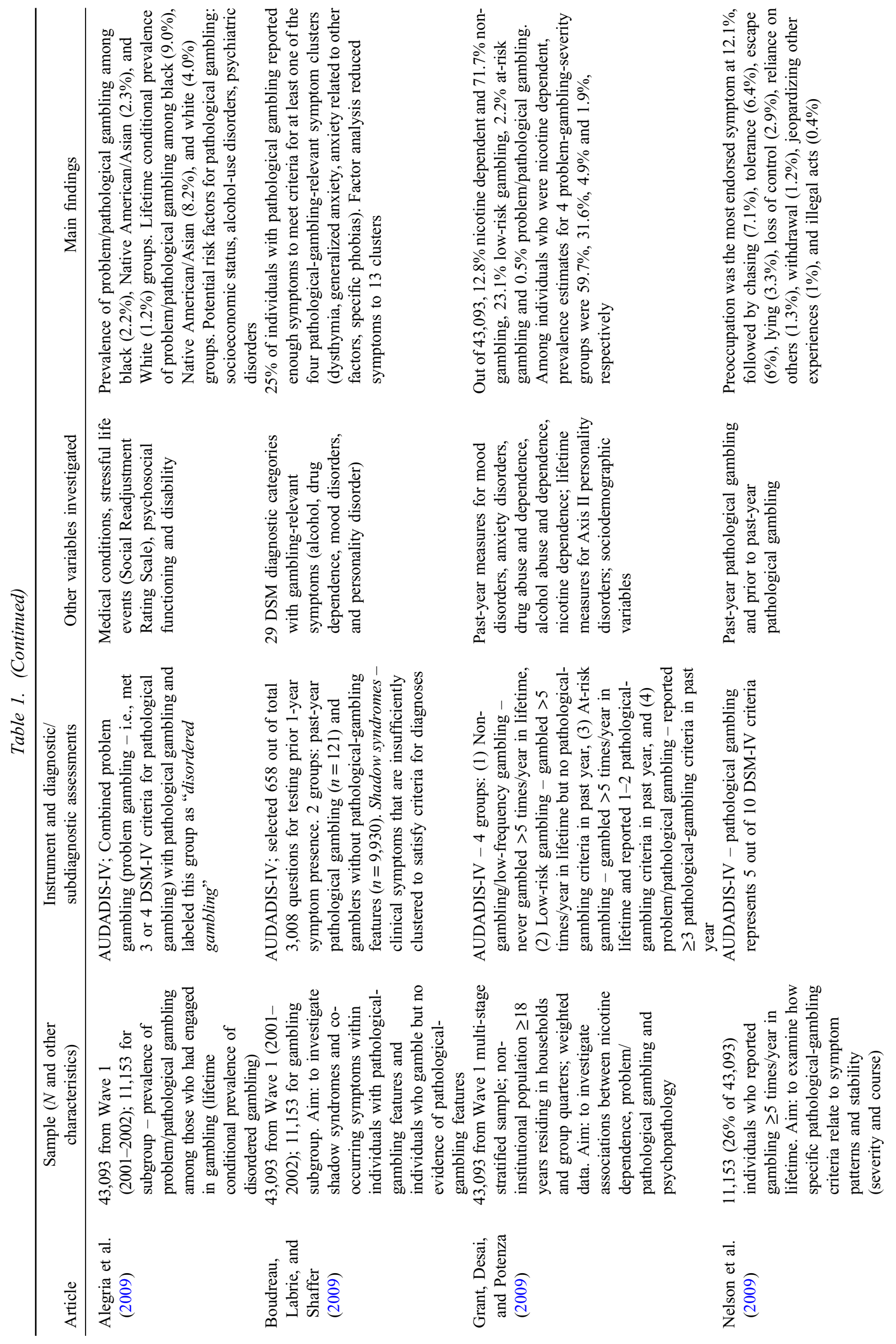



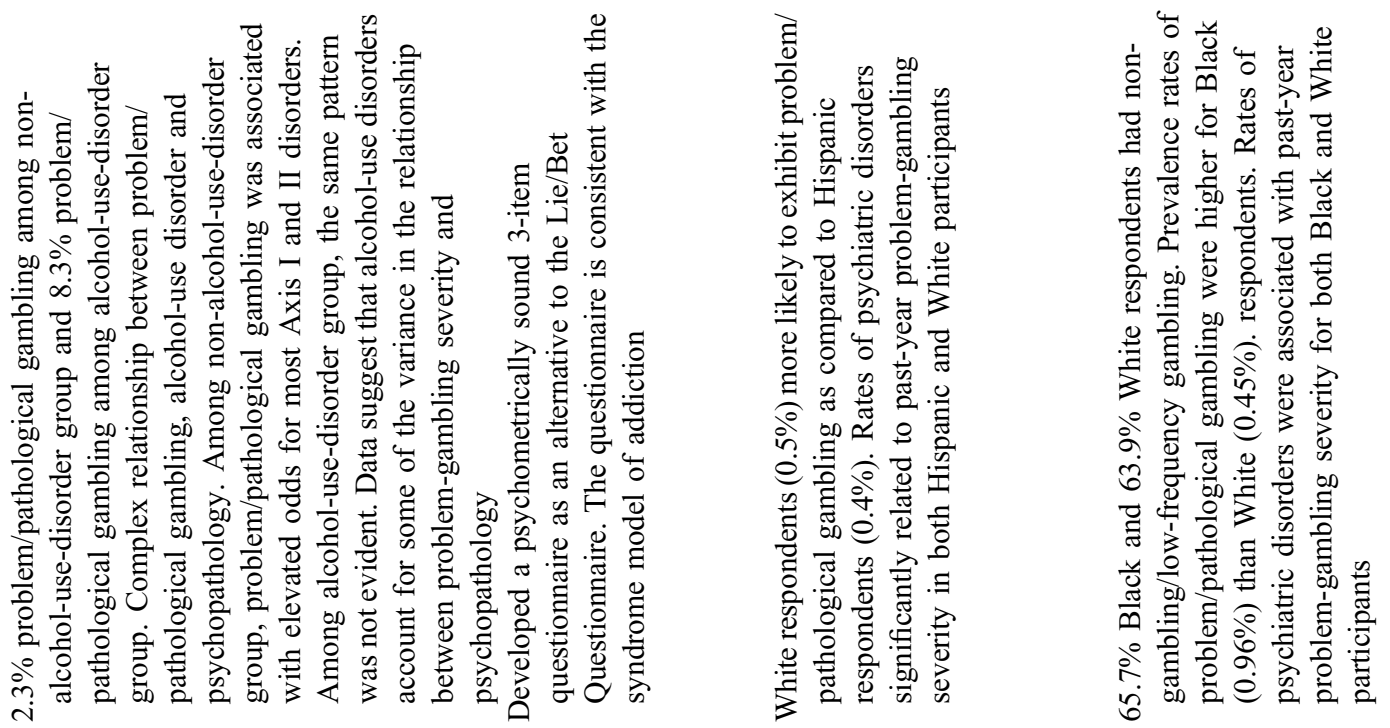

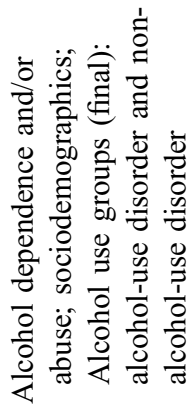

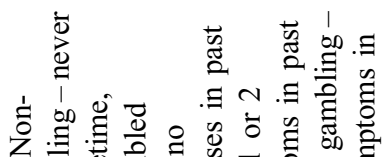

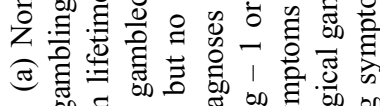

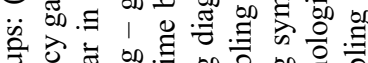

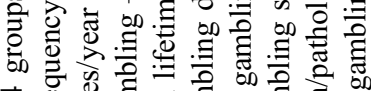

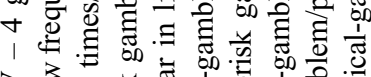

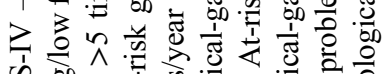

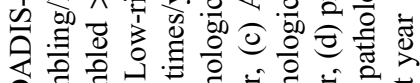

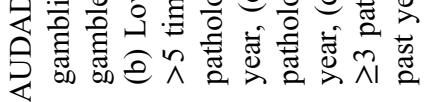

客

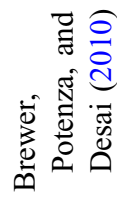
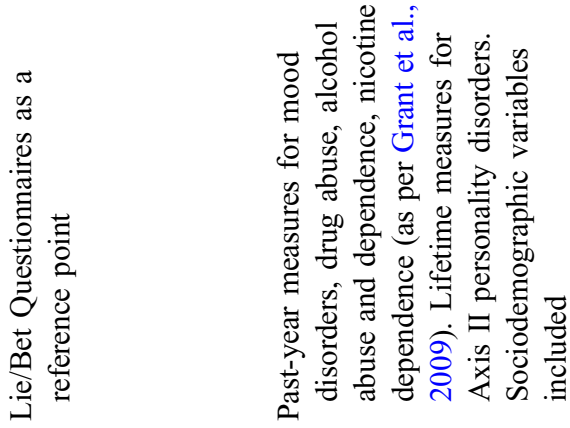
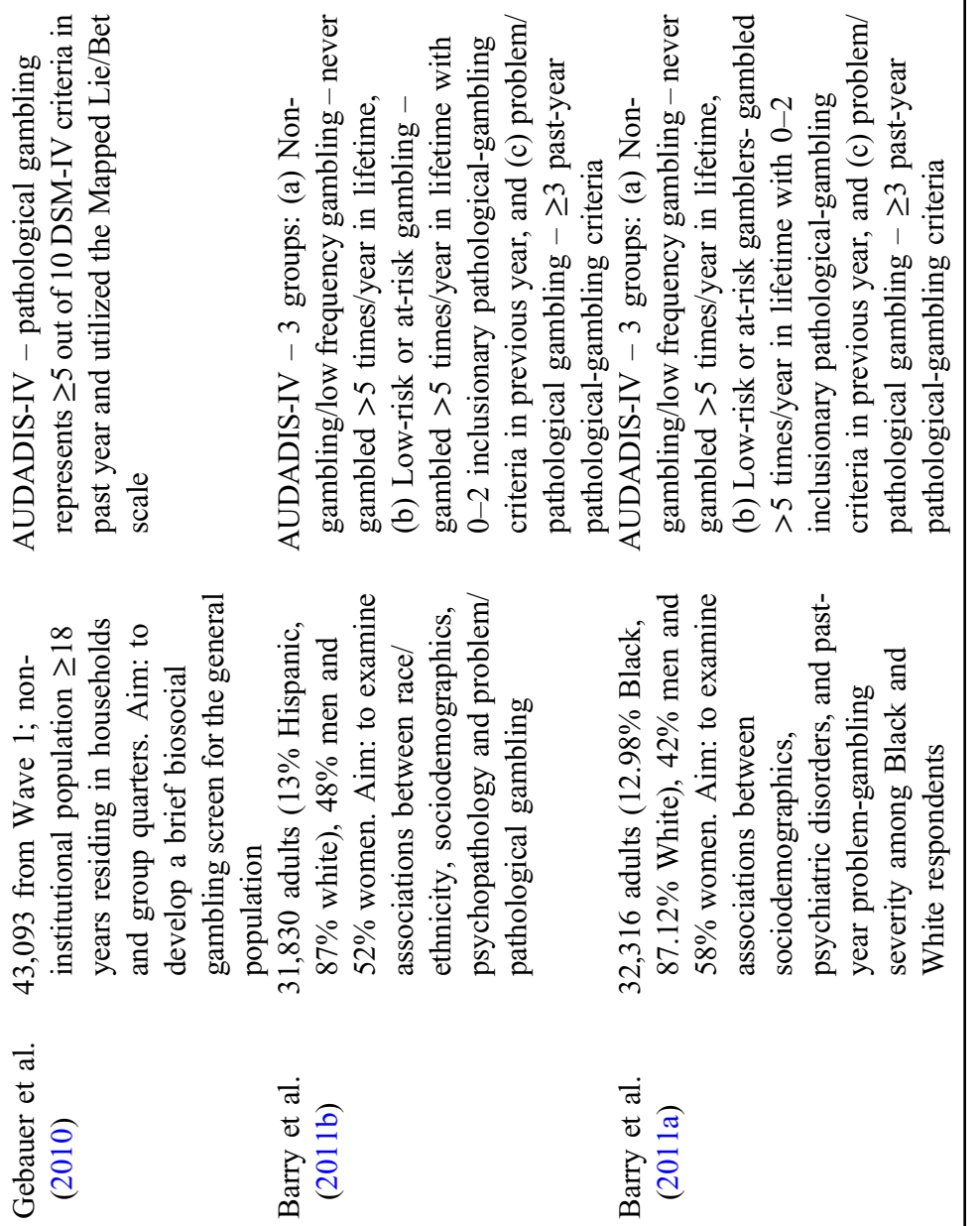

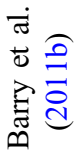

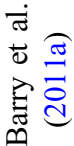




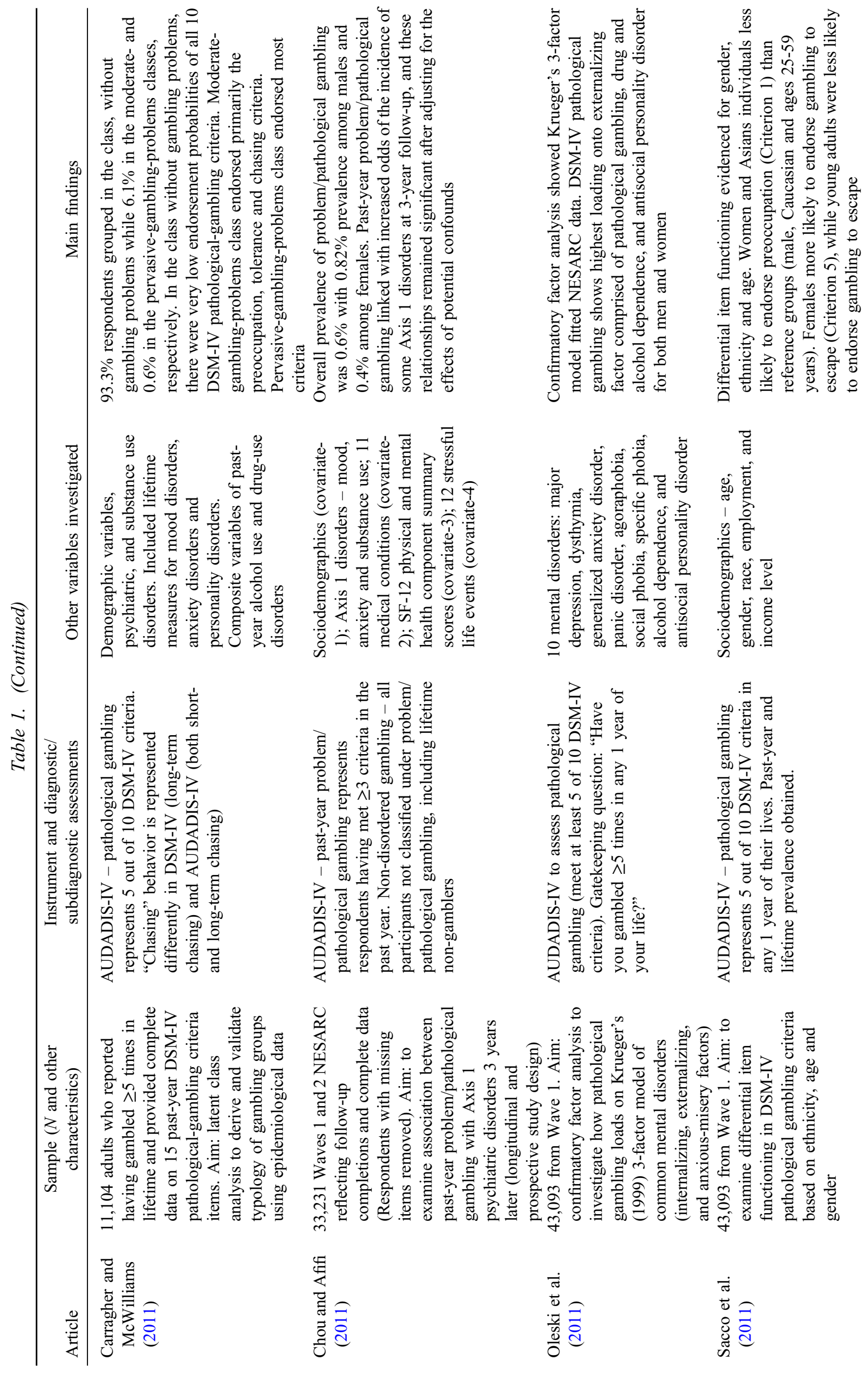




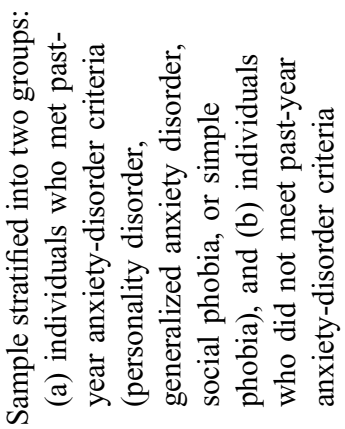

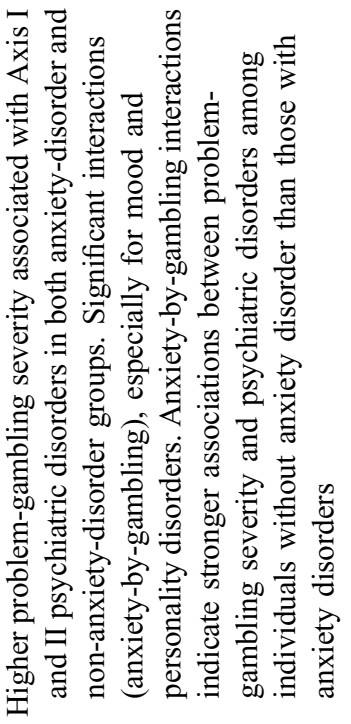

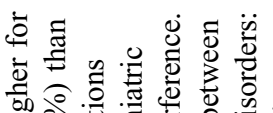

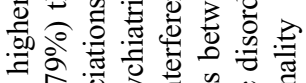

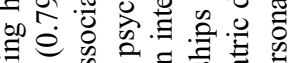

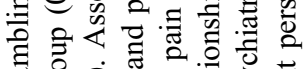

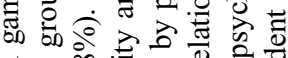

चु

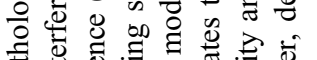

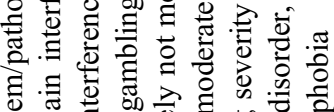

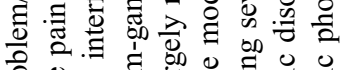

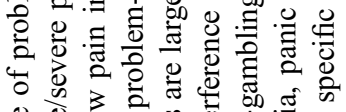

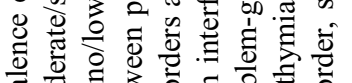

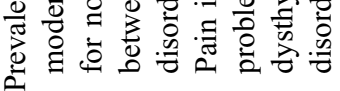

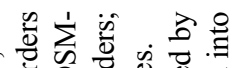

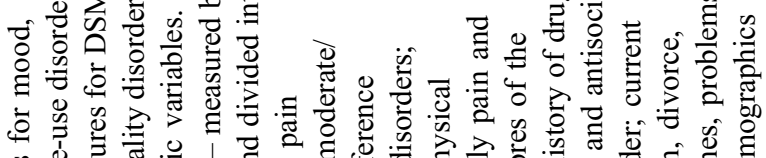

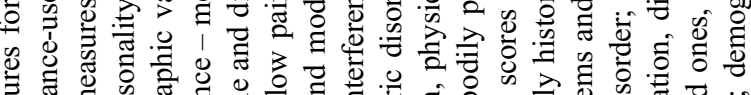

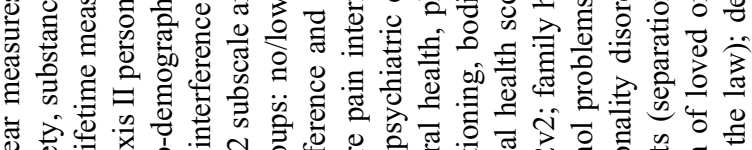

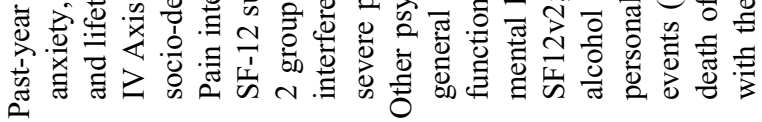

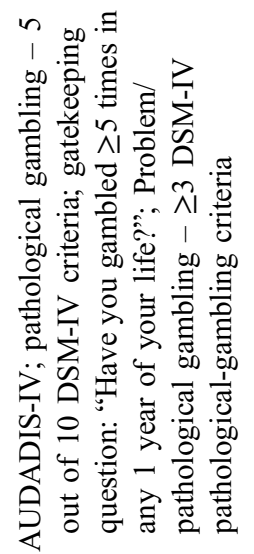

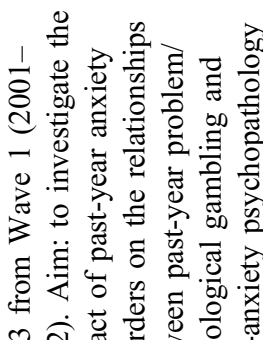

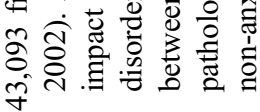

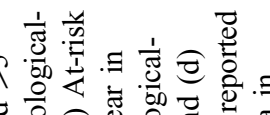

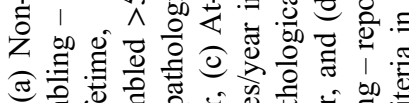

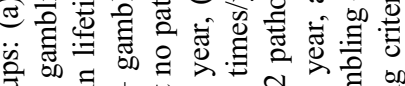

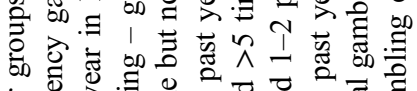

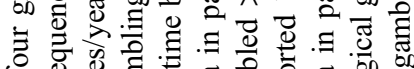

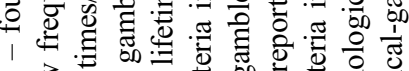

离 $2 \frac{0}{2}$

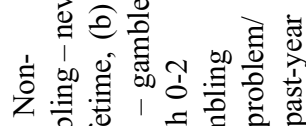

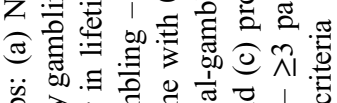

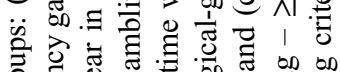

के

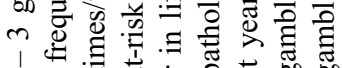

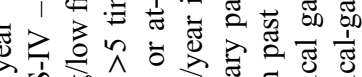
光

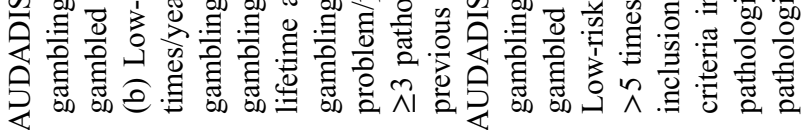

$\frac{\pi}{\pi}$
$\frac{0}{0}$
$\frac{0}{0}$
$\frac{0}{0}$
灵

3 ธิ อ

○. 을

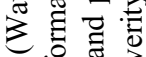

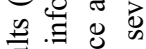

渵

ᄅृ

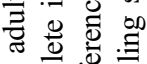

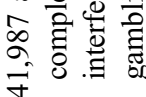

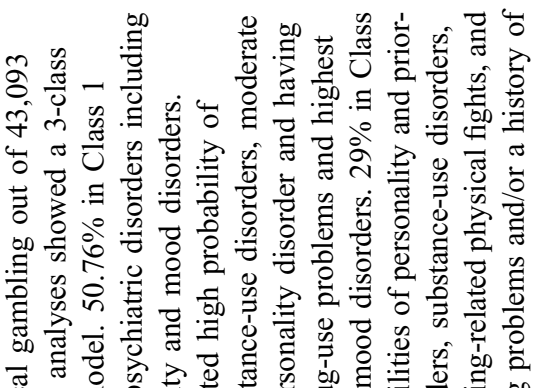

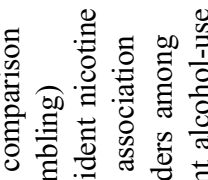

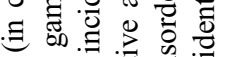

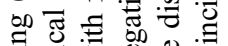

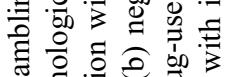

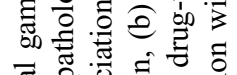

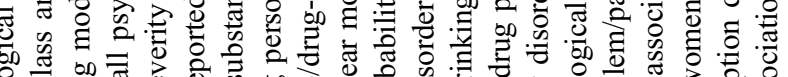

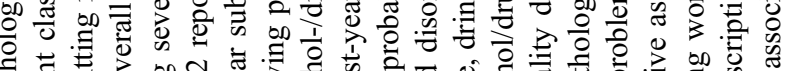

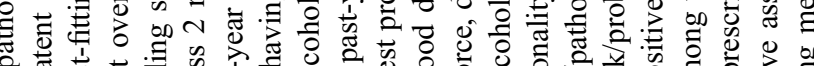

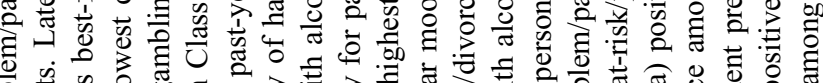

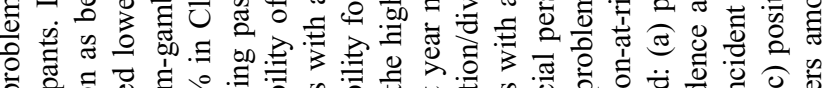

ప.

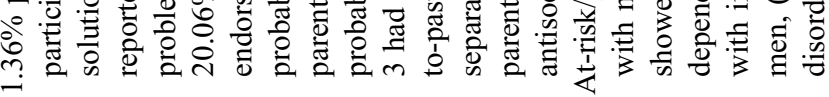
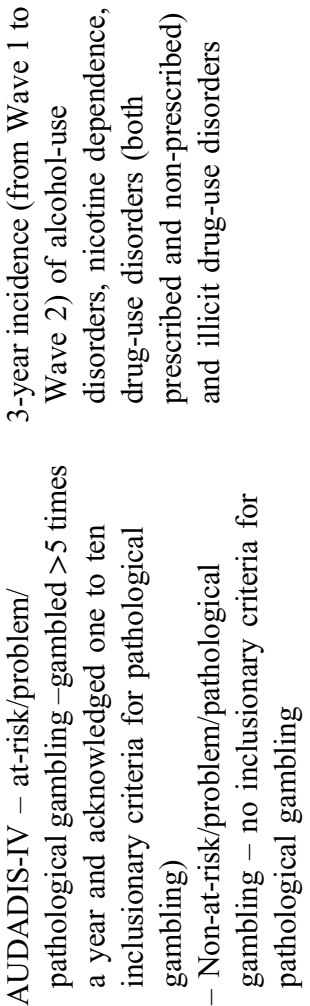

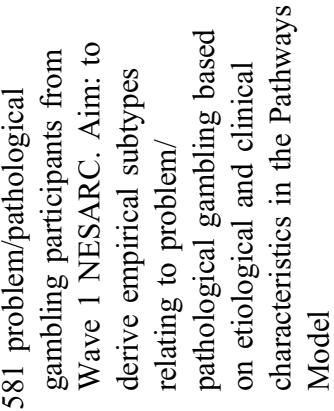

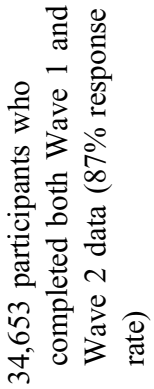

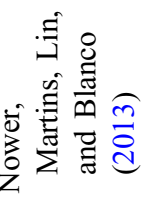

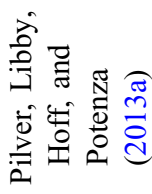




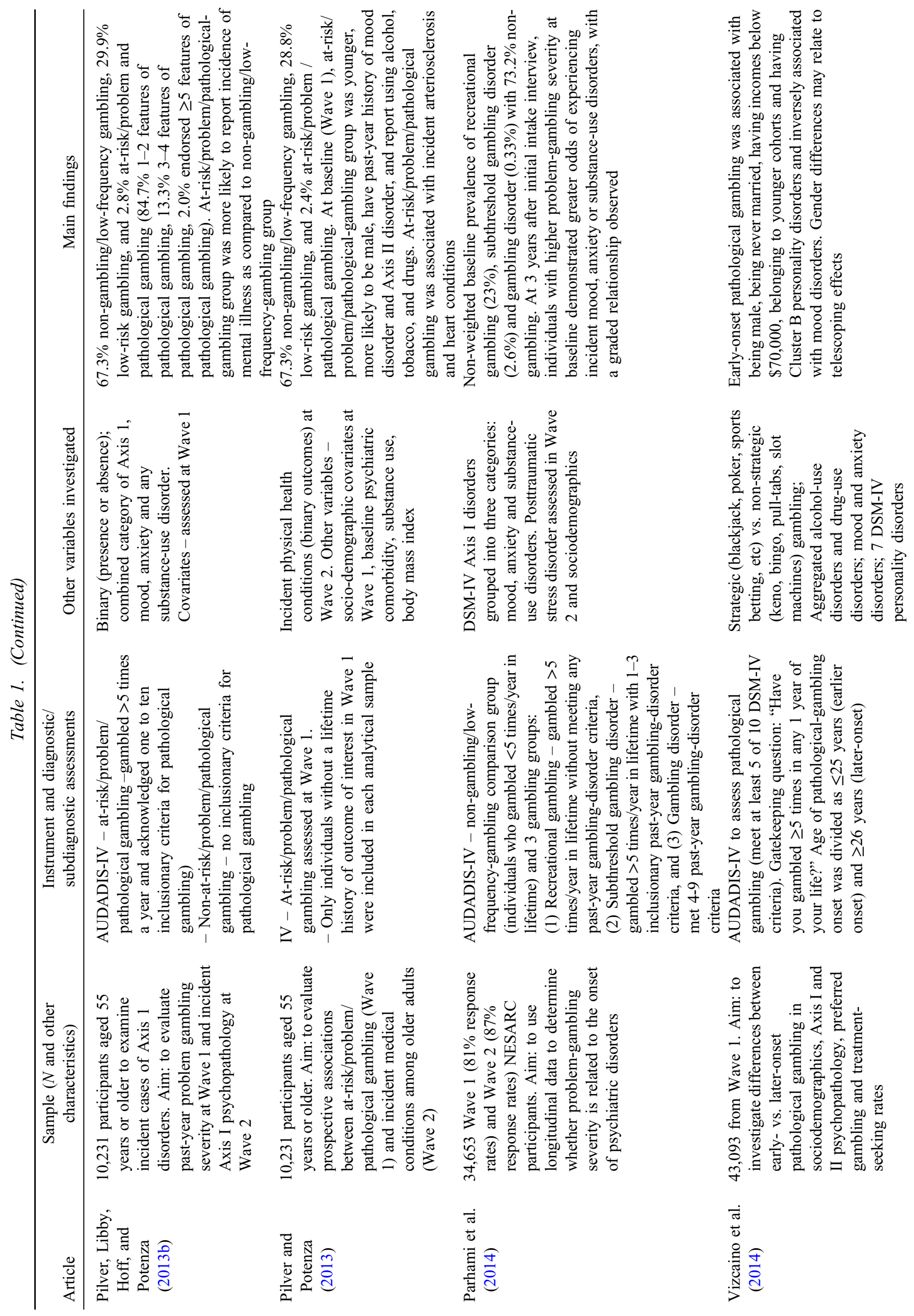



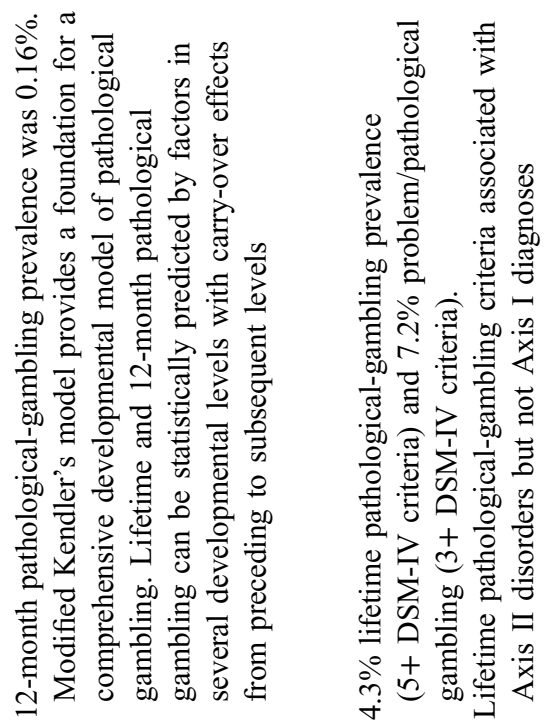

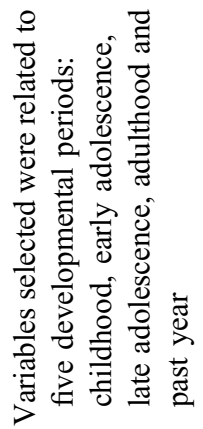

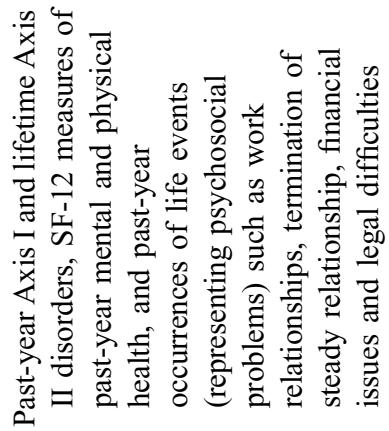

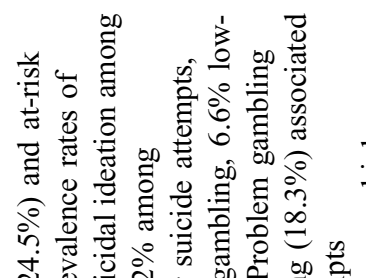

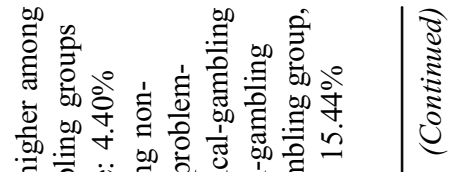

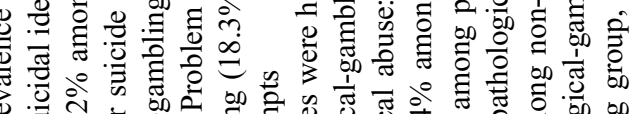

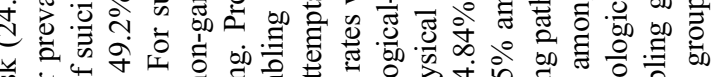
芸

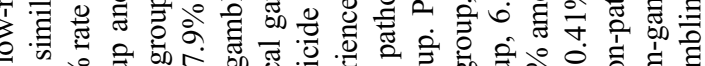

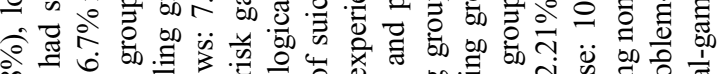

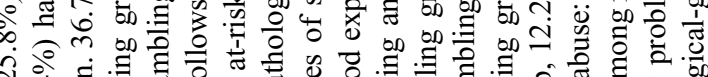
ปे

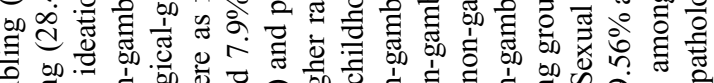

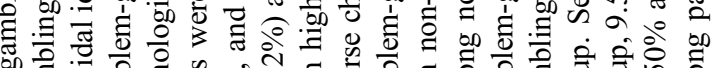

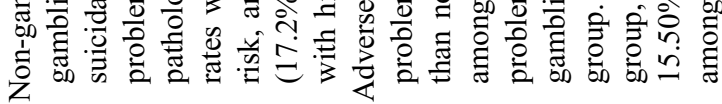

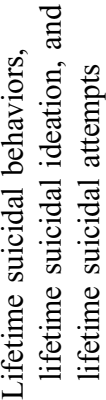

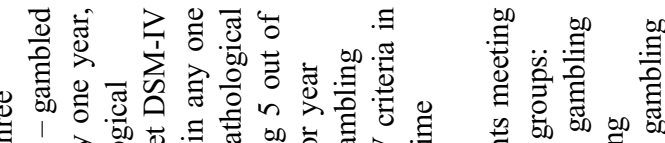

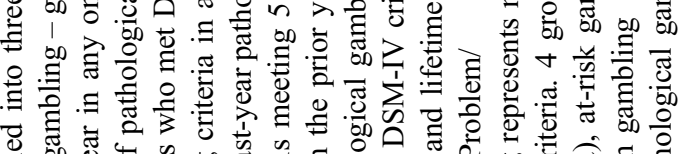

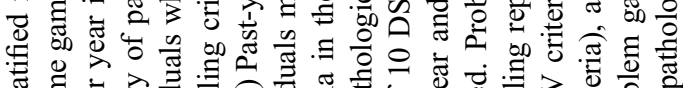

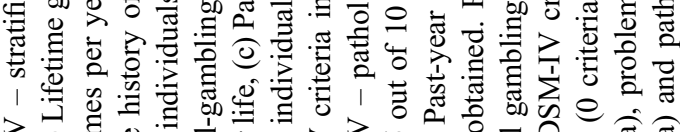

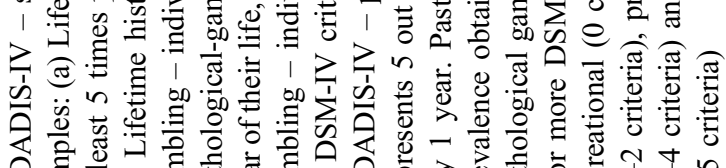

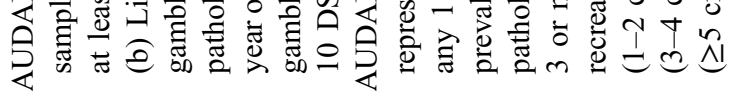

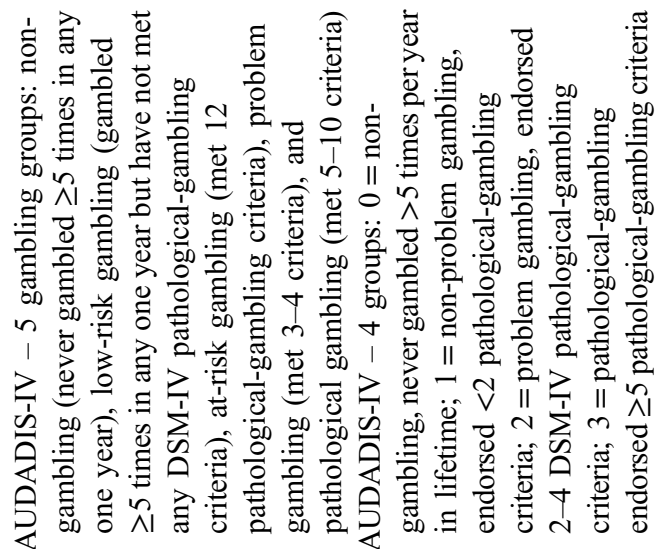

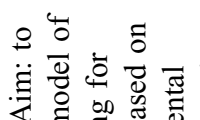

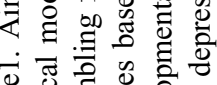

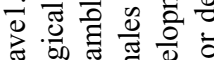

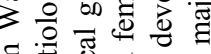

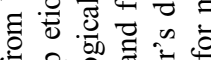

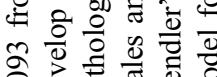

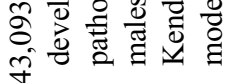

$$
\text { त }
$$

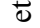

章 $\frac{\pi}{0}$

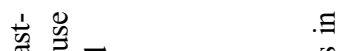

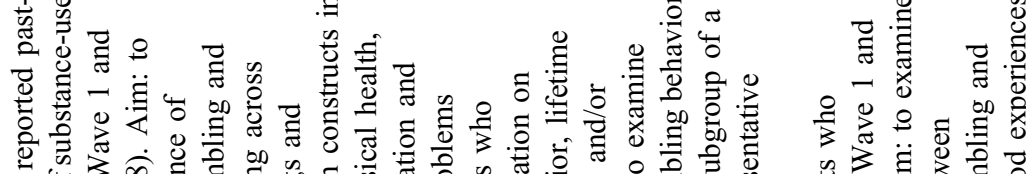

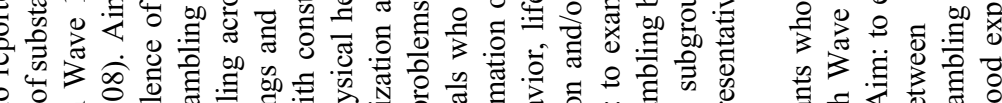

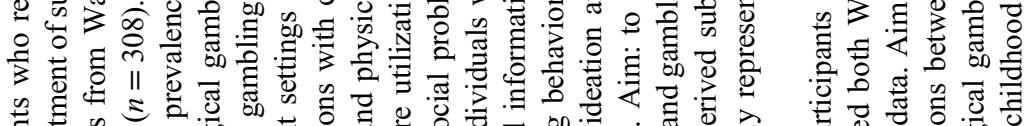

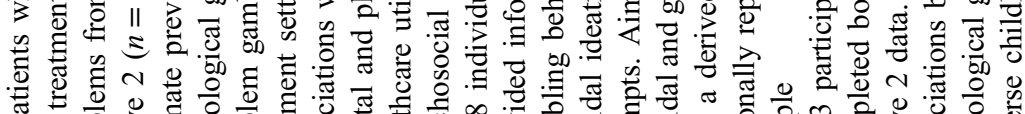

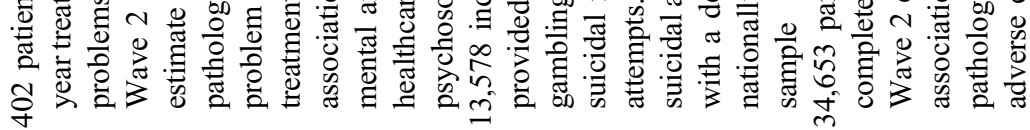

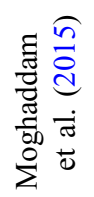

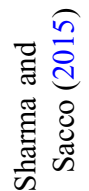




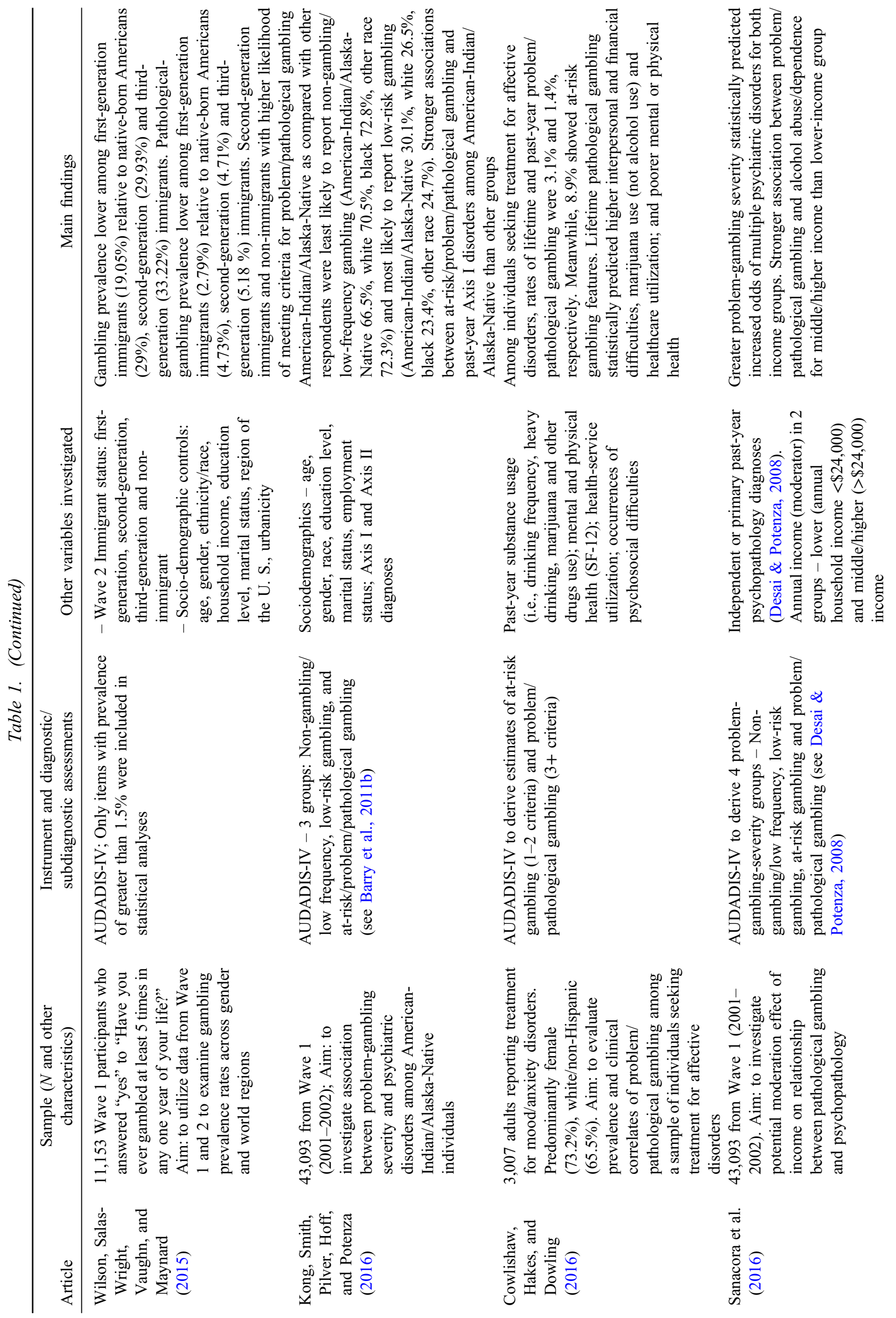



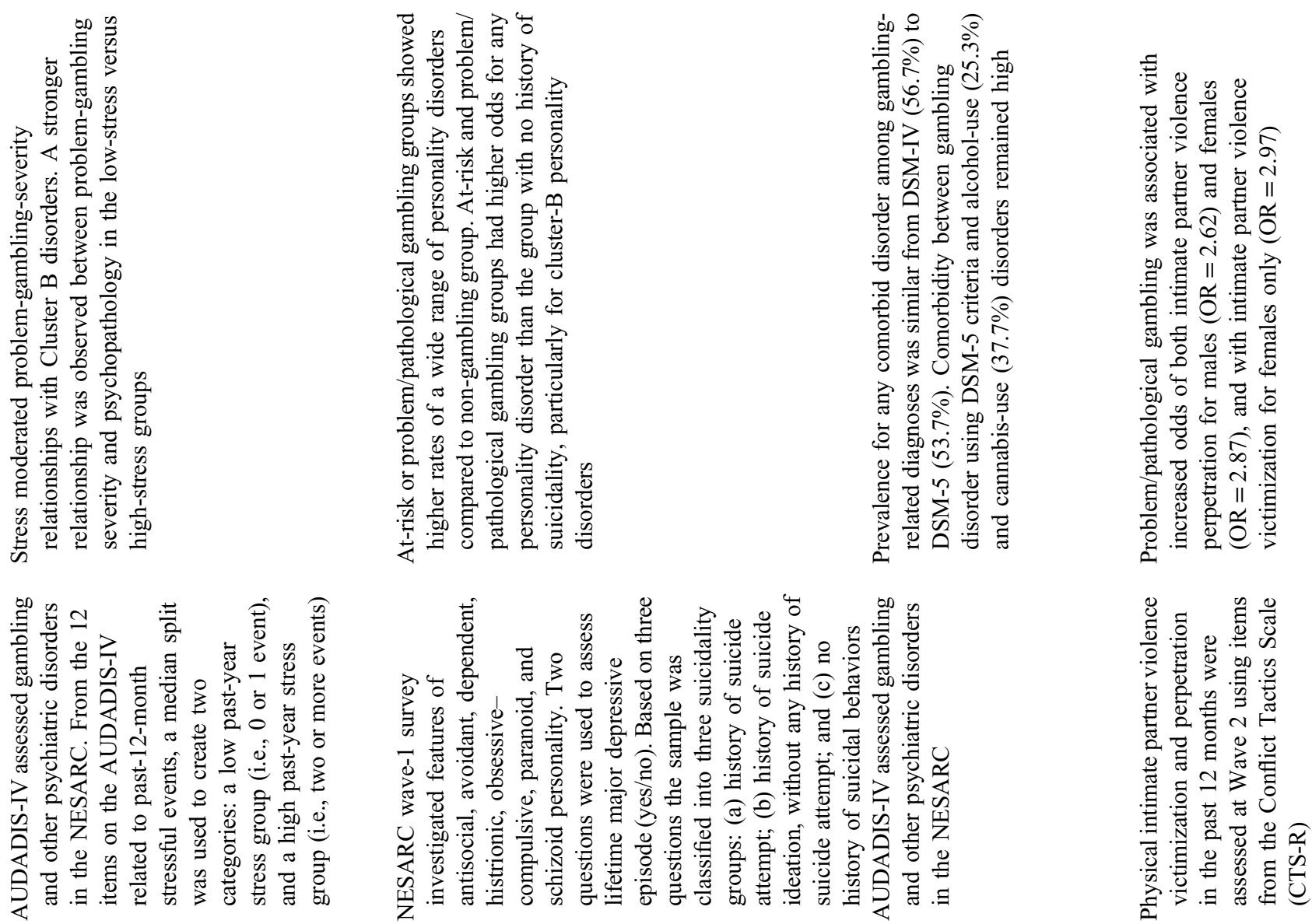

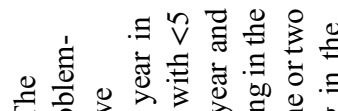

\section{-}

苂

它

$\Xi \quad \overline{\frac{\pi}{\pi}}$

密

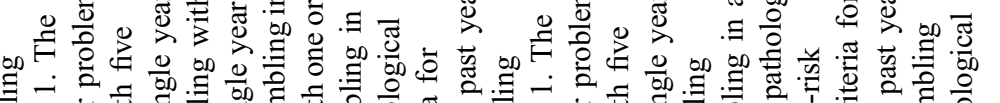

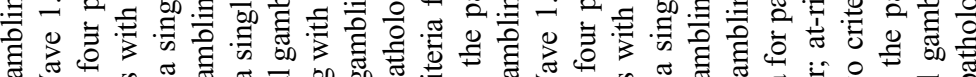

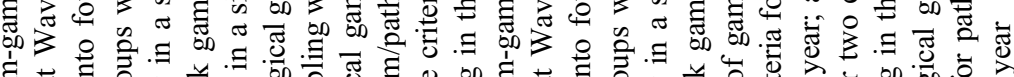

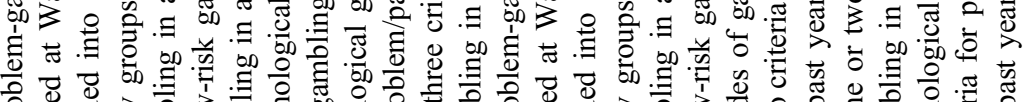

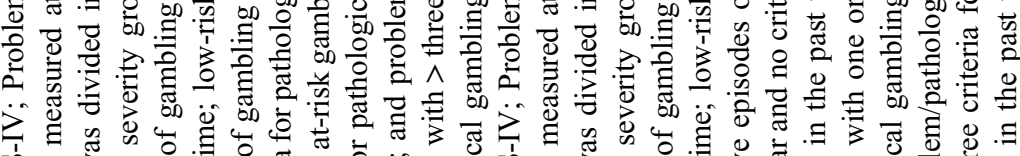

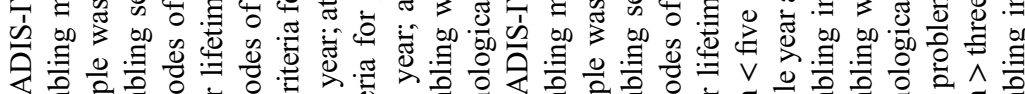

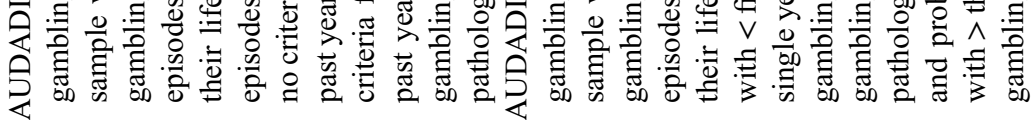

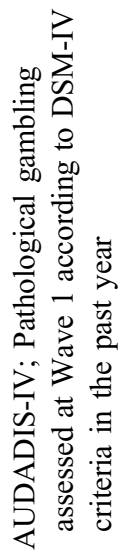

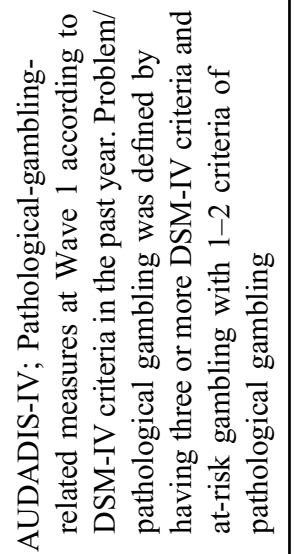

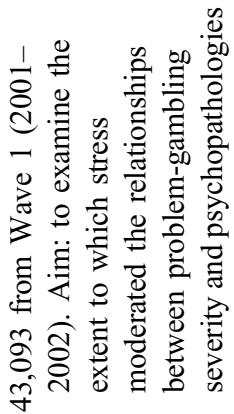

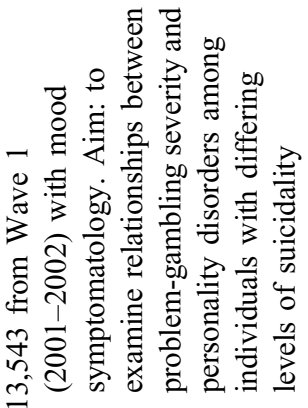

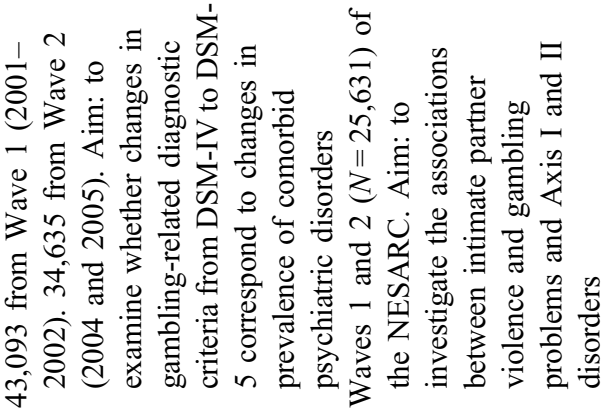

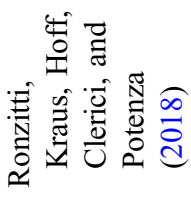

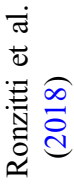

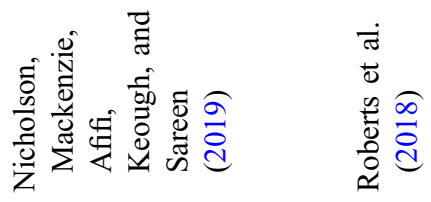




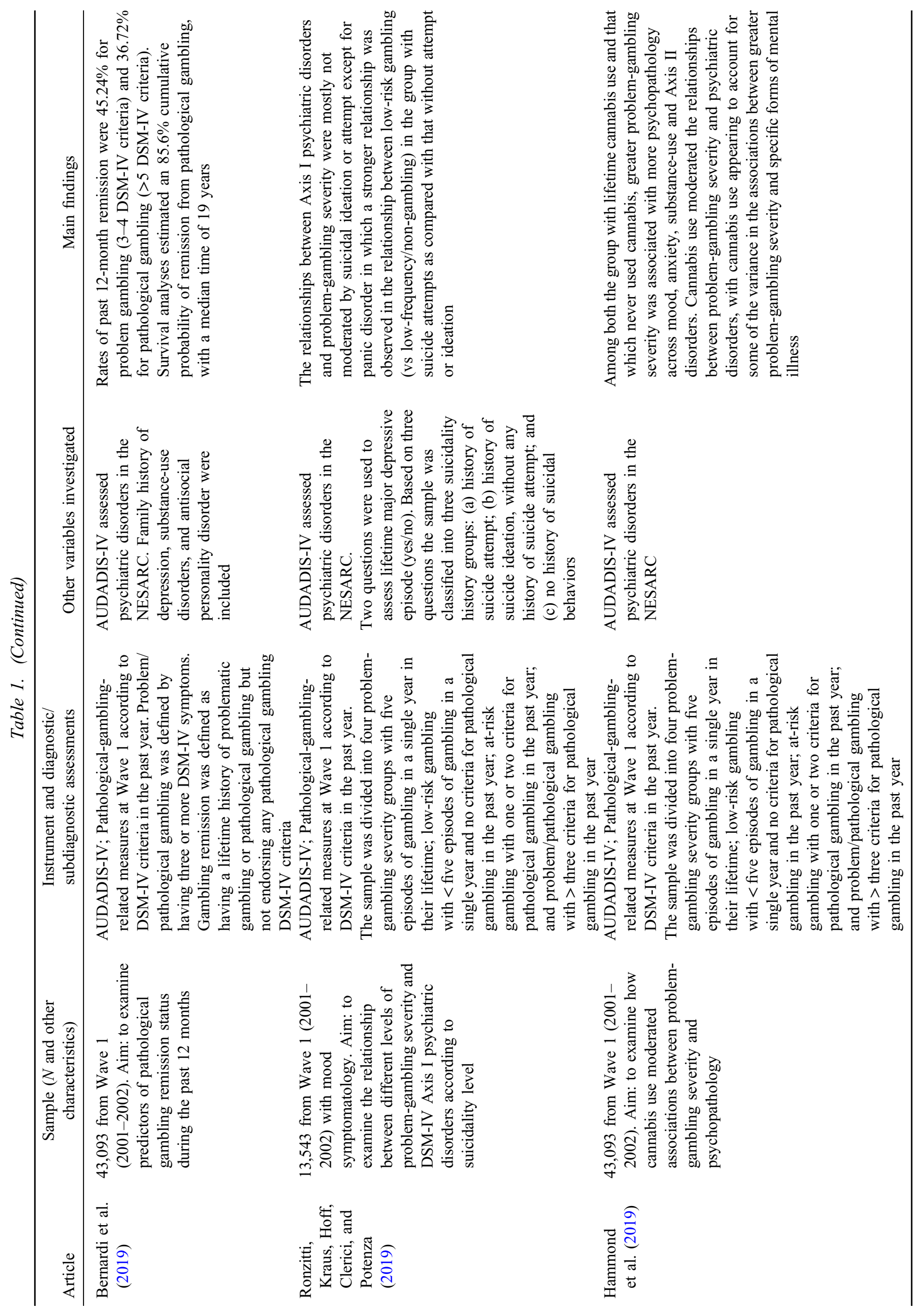




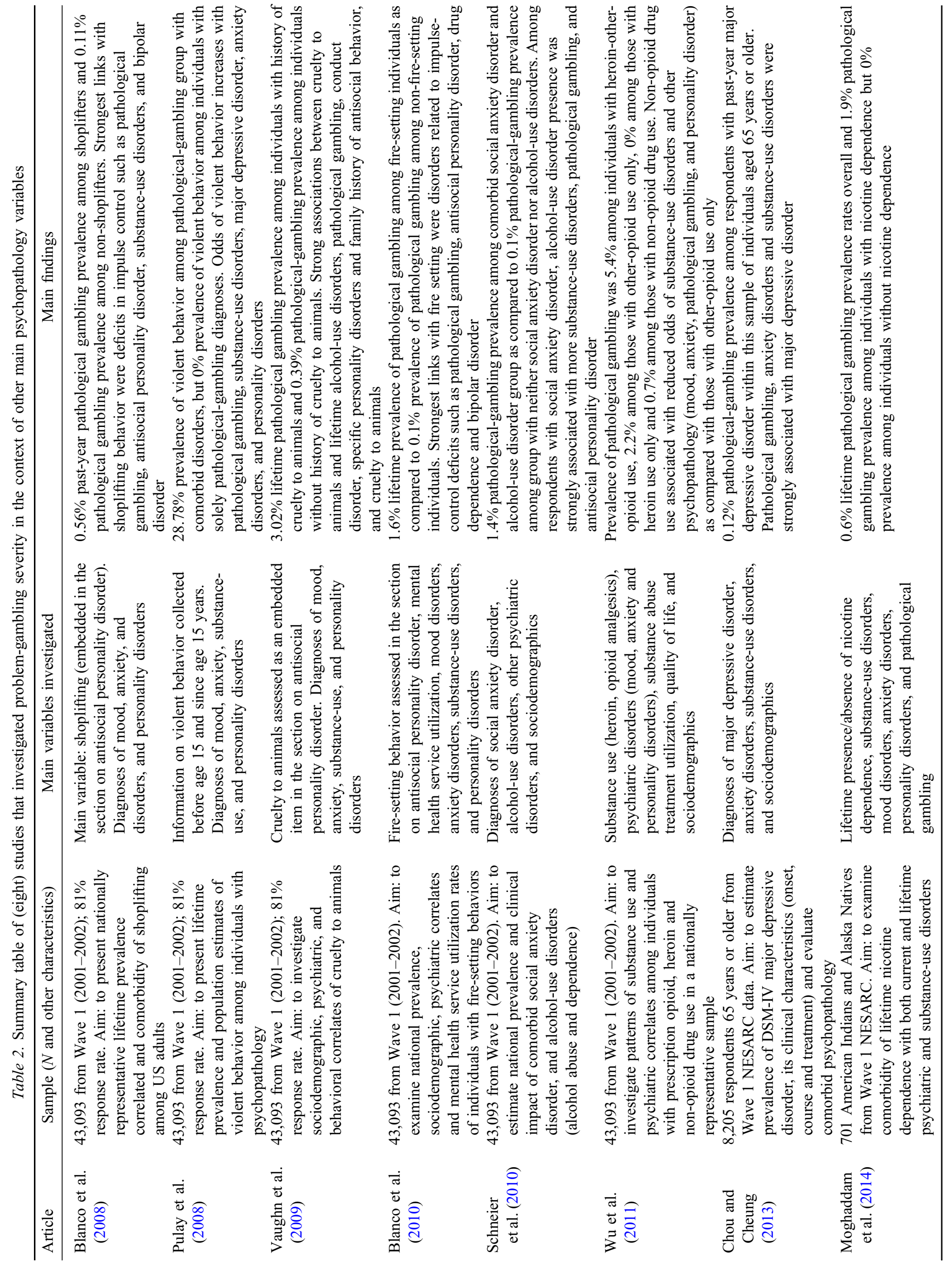


was conducted at Wave 1 with the psychometrically validated AUDADIS-IV instrument, which is an extensive semi-structured diagnostic interview conducted by trained lay interviewers to assess lifetime and past-year psychiatric disorders and related measures (Grant \& Dawson, 2006). Other psychiatric disorders assessed in the AUDADIS-IV included: (a) five mood disorders/features - major depressive disorder, bipolar I and II disorders, dysthymia, and hypomania; (b) four/five anxiety disorders - panic disorder with and without agoraphobia, social phobia, specific phobia, and generalized anxiety; and (c) seven personality disorders - avoidant, dependent, obsessivecompulsive, paranoid, schizoid, histrionic, and antisocial disorders.

There are several distinctions between Waves 1 and 2 estimates - Wave 1 data captured respondents' lifetime and past-year experiences, whereas Wave 2 focused on priorto-past-year (since Wave 1) and past-year psychopathology experiences. Additional assessments in Wave 2 included: (a) classifications for several psychiatric disorders posttraumatic stress disorder, attention-deficit hyperactivity disorder, and narcissistic, borderline, and schizotypal personality disorders; (b) psychosocial indicators - sexual orientation, adverse childhood events, childhood and partner abuse, social integration, and acculturation; and (c) perceived experiences of discrimination.

Definitions of problem-gambling-related behaviors have changed over time in accordance with empirical findings and progression of DSM and non-DSM efforts like the Research Domain Criteria movement - with both categorical and continuum conceptualizations of problem-gambling severity (from non-gambling/low frequency gambling to pathological gambling). Although several different thresholds and definitions have been utilized (see Table 1), pathological gambling in DSM-IV was defined by meeting 5 or more of 10 criteria, whereby 15 symptom items operationalized the 10 pathological-gambling criteria in the assessment used in the NESARC (Blanco et al., 2006; Petry et al., 2005). Meanwhile, meeting three to four criteria has been termed by some authors as experiencing problem gambling - defined by some as excessive gambling linked with impaired inter- and intra-personal functioning but not meeting the minimum threshold of pathological-gambling criteria in DSM-IV (Loo, Raylu, \& Oei, 2008). The gatekeeping question in the AUDADIS-IV was "Have you gambled 5 or more times in any one year of your life?" and respondents who answered "No" to this question were classified as low-frequency/non-gambling, while past-year recreational gambling involved answering "Yes" but met no more than two pathological-gambling criteria in the previous year (Desai et al., 2007; Pietrzak et al., 2007). Some authors have further separated recreational gambling into low-risk and at-risk groups, with the former meeting no criteria and the latter 1-2 criteria for pathological gambling (Desai \& Potenza, 2008; Grant, Desai, \& Potenza, 2009). Some studies combined problem gambling and pathological gambling into a single group to increase statistical power and labeled it as problem/pathological gambling, where respondents met three or more pathological-gambling criteria (Barry, Pilver, Hoff, \& Potenza, 2013; Barry, Stefanovics, Desai, \& Potenza, 2011a, 2011b; Desai \& Potenza, 2008;
Giddens, Stefanovics, Pilver, Desai, \& Potenza, 2012; Grant et al., 2009). Most studies distinguished between lifetime gambling and past-year pathological gambling, and presented weighted prevalence estimates for the population of interest.

\section{Psychometric developments and measurement analyses with NESARC data}

There were five gambling-related NESARC studies that developed research questions on model testing, symptom patterns, differential item functioning, and/or scale development (i.e., Boudreau et al., 2009; Gebauer, LaBrie, \& Shaffer, 2010; Nelson, Gebauer, LaBrie, \& Shaffer, 2009; Sacco, Torres, Cunningham-Williams, Woods, \& Unick, 2011; Strong \& Kahler, 2007). Nationally representative data are an important resource from which symptom classifications and assessment perspectives can be reviewed in a reliable manner. Strong and Kahler (2007) evaluated the DSM-IV pathological-gambling criteria - using 11,153 lifetime gambling Wave 1 data points - for its unidimensionality and symptom patterns. Factor analysis results revealed that pathological gambling symptoms fit a unidimensional Rasch model, which confirms the validity of using a total pathological gambling score to represent symptom patterns variations in problem-gambling severity. Extending these findings, Sacco et al. (2011) explored the presence of differential item functioning in DSM-IV pathological-gambling criteria stratified by age, gender, and ethnicity/race using Multiple-Indicator Multiple-Cause modeling and found evidence for differential item functioning for these sociodemographic variables. Specifically, women and Asians were less likely than reference groups (male, Caucasian, ages 25-29 years) to endorse preoccupation, and women were more likely to report gambling to escape, while young adults were less likely to report gambling to escape (see Table 1 for additional details).

Utilizing a similar sampling method and 658 out of 3,008 AUDADIS-IV questions, Boudreau et al. (2009) investigated the presence of shadow symptoms (e.g., anxiety and phobias) among individuals with and without past-year pathological gambling behavior. Findings suggested that shadow symptoms were associated with pathological gambling diagnoses, providing support for a need to develop treatment around multi-faceted symptomology instead of categorical diagnostic classification (see Table 1 for additional details). Following from this study, Nelson et al. (2009) found that pathological gambling symptom patterns changed as symptom count increased and differed between timeframes. Preoccupation, chasing losses, tolerance, and gambling to escape were the top four most endorsed criteria, whereas illegal acts were both rare, unstable, and arguably the least useful discriminant criteria (Nelson et al., 2009). As preoccupation is both prevalent and stable across timeframes, it is a useful gateway question in clinical settings. Building on this symptom analysis research, Gebauer et al. (2010) validated a 3-item brief biosocial gambling screen applicable for use among community populations and treatment-seeking groups. The Brief Biosocial Gambling Screen, which is an alternative to the Lie/Bet Questionnaire, showed high sensitivity and specificity, with an item from 
each of the three theoretical addiction syndrome domains neuroadaptation, psychosocial characteristics, and negative social consequences of gambling.

\section{Prevalence of pathological gambling}

The lifetime pathological gambling prevalence estimate among NESARC Wave 1 respondents was $0.42 \%(0.64 \%$ men and $0.23 \%$ women), $N=43,093$ (Petry et al., 2005). Past-year pathological gambling prevalence was $0.16 \%$ (Blanco et al., 2015), but when restricted to sample of lifetime gamblers, past-year pathological gambling rates were $1.92 \%$ among men and $1.05 \%$ among women (Blanco et al., 2006). Morasco et al. (2006) reported lifetime prevalence rates of $0.42 \%$ for pathological gambling, with $25.84 \%$ exhibiting at-risk gambling and $72.84 \%$ exhibiting low-risk gambling among gamblers. Past-year problem/ pathological gambling rates were reported to be $0.70 \%$ in men and $0.40 \%$ in women (Desai \& Potenza, 2008). Among 33,231 complete data from Waves 1 and 2, problem/ pathological gambling rate was $0.60 \%$ with $0.82 \%$ prevalence among males and $0.40 \%$ among females (Chou \& Afifi, 2011). When categorized according to immigrant status, pathological gambling prevalence rate was highest for third-generation immigrants $(5.18 \%)$, followed by native-born Americans (4.73\%) and second-generation immigrants $(4.71 \%)$, while pathological gambling prevalence was lowest for first-generation immigrants at $2.79 \%$ (Wilson et al., 2015).

Adults aged 40-64 years reported weighted prevalence estimates of $0.30 \%$ for problem/pathological gambling and $30.80 \%$ for recreational gambling, while older individuals aged more than 64 years reported similar rates - problem/ pathological gambling $(0.30 \%)$ and recreational gambling (28.70\%; Desai et al., 2007). Comparable estimates were found in another study (Table 1), which suggested that recreational gambling was relatively common among older adults (Pietrzak et al., 2007). Further evaluations on Wave 1 pathological gambling and Wave 2 psychopathology revealed that baseline prevalence rates among older adults aged 55 years or more were $2.80 \%$ for at-risk/problem/ pathological gambling and $29.90 \%$ for low-risk gambling (Pilver et al., 2013b). Respondents with past-year major depressive disorder aged 65 years or older reported a modest pathological gambling prevalence of $0.12 \%$ (Chou \& Cheung, 2013).

As pathological gambling rarely exists in isolation, prevalence estimates for gambling groups (non-gambling/low frequency, low-risk, at-risk, and problem/pathological gambling) among nicotine-dependent individuals were 59.70\%, $31.60 \%, 4.90 \%$, and $1.9 \%$, respectively (Grant et al., 2009). In a sample of 701 American Indians, pathological gambling prevalence was higher among individuals with (1.90\%) than without ( $0 \%$ ) nicotine dependence (Moghaddam, Dickerson, Yoon, \& Westermeyer, 2014). Meanwhile, problem/ pathological gambling rate was higher at $8.30 \%$ among individuals with alcohol abuse and/or dependence, and lower at $2.30 \%$ among non-alcohol-use-disorder group (Brewer, Potenza, \& Desai, 2010). Analyses of 402 patients who reported past-year treatment of substance-use problems revealed prevalence estimates of $4.30 \%$ of lifetime pathological gambling and $7.20 \%$ of problem gambling (Cowlishaw \& Hakes, 2015). Hammond et al. (2019) also found that cannabis use moderated the relationships between problem-gambling severity and psychiatric disorders, with cannabis use appearing to account for some of the variance in the associations between greater problem-gambling severity and specific forms of mental illness.

Meanwhile, data from 3,007 treatment-seeking individuals with affective disorders showed comparably lower rates $3.1 \%$ lifetime pathological gambling and $1.4 \%$ past-year problem gambling (Cowlishaw et al., 2016). Pathological gambling prevalence was higher among individuals (1.40\%) with comorbid social anxiety disorder and an alcohol-use disorder, as compared with individuals $(0.10 \%)$ who were diagnosed neither with social anxiety disorder nor an alcohol-use disorder (Schneier et al., 2010). In another study on specific drug use, pathological gambling prevalence was highest among heroin-other-opioid-using individuals (5.40\%) and other-opioid-only-using individuals (2.20\%), as compared with heroin-using $(0 \%)$ and non-opioid-using $(0.70 \%)$ individuals (Wu, Woody, Yang, \& Blazer, 2011). Nicholson et al. (2019) recently reanalyzed Wave 1 data to examine whether changes in gambling-related diagnostic criteria from DSM-IV to DSM-5 would correspond to changes in prevalence of psychiatric disorders among those with pathological gambling versus gambling disorder. Prevalence rates for any comorbid disorders among individuals with pathological gambling versus gambling disorder remained similar $(56.7 \%$ vs. $53.7 \%)$ and the highest cooccurring substance-use disorders were alcohol (25.3\%) and cannabis $(37.7 \%)$.

The AUDADIS-IV captured information on antisocialpersonality disorder that provided an avenue to estimate pathological-gambling prevalence among individuals with the presence of specific antisocial-personality disorder behaviors. Past-year pathological-gambling prevalence was reportedly higher among shoplifters $(0.56 \%)$, as compared with non-shoplifters at $0.11 \%$ (Blanco et al., 2008). Similarly, lifetime pathological gambling prevalence was evident among individuals who reported animal cruelty behavior (3.02\% with vs. $0.39 \%$ without) and fire-setting behavior $(1.60 \%$ with vs. $0.10 \%$ without) (Blanco et al., 2010; Vaughn et al., 2009). In addition, violent behavior was more prevalent among individuals with pathological gambling with comorbid disorders $(28.78 \%)$, such as alcohol-use and mood disorders, than among those with solely pathological gambling (0\%) (Pulay et al., 2008). In addition, Ronzitti et al. (2018) found that at-risk/problem/ pathological gambling had a higher odds ratio for any personality disorder in the group with no history of suicidality, particularly for cluster-B personality disorders.

Prevalence for problem/pathological gambling was higher for a group with moderate/severe pain interference $(0.79 \%)$ than for a group with no/low pain interference $(0.48 \%)$, while similar prevalence patterns were observed for low-risk-gambling or at-risk-gambling groups (Barry et al., 2013). Both problem gambling and pathological gambling groups as compared with non-gambling or lowrisk gambling groups had significantly higher prevalence estimates of suicidal ideation $(36.70 \%, 49.20 \%)$ and attempts $(17.20 \%, 18.30 \%)$, respectively (Moghaddam, 
Yoon, Dickerson, Kim, \& Westermeyer, 2015). Furthermore, adverse childhood experiences rates were higher for problem-gambling and pathological-gambling groups than for a non-gambling group (Sharma \& Sacco, 2015). Specifically, rates of physical and sexual abuse were $6.95 \%$ and $15.50 \%$ among individuals with problem gambling and $12.21 \%$ and $15.44 \%$ among those with pathological gambling, respectively.

\section{Gambling subtypes and model testing}

Six studies included in this systematic review utilized NESARC data to evaluate theoretical models of pathological gambling, identify gambling subtypes, or distinguish between individuals with early- versus later-onset pathological gambling. Individuals with early-onset pathological gambling were more likely than those with later-onset pathological gambling to be male, be never married, have incomes below $\$ 70,000$, belong to younger cohorts and have Cluster B personality disorders, and were less likely to have mood disorders (Vizcaino, Fernandez-Navarro, Petry, Rubio, \& Blanco, 2014). Bernardi et al. (2019) examined predictors of pathological gambling remission during the past 12 months and found that an $85.6 \%$ cumulative probability of remission from pathological gambling with a median time of 19 years. Carragher and McWilliams (2011), through latent class analysis on 11,104 lifetime gamblers, empirically derived three typology of gamblers groups with no, moderate, and pervasive gambling problems - based on endorsements of past-year DSM-IV pathological-gambling criteria. While the first group (no gambling problems) showed very low endorsement probabilities across all criteria, the second group (moderate) endorsed primarily preoccupation, tolerance, and chasing criteria, and the third group (pervasive) endorsed most criteria. Also using latent class analysis, but this time with 581 individuals with problem/pathological gambling, Nower et al. (2013) derived three problem-gambling subtypes/classes based on etiological and clinical characteristics in a Pathways Model. Individuals in Class 1 $(50.76 \%)$ showed lowest overall psychiatric disorders, while those in Class $2(20.06 \%)$ showed high probability of endorsing past-year substance-use disorders and mood disorders. Finally, those in Class 3 had the highest probability of personality disorders, substance-use disorders, and separation/divorce, among other concerns (see Table 1 for additional details).

In the first attempt of examining model fit using NESARC Wave 1 data, findings from confirmatory factor analysis showed that pathological gambling loaded highest on the externalizing factor of Krueger's (1999) threefactor model of common mental disorders (Oleski, Cox, Clara, \& Hills, 2011). The externalizing factor composed of pathological gambling, substance dependence, and antisocial-personality disorder for both genders. Blanco et al. (2015) developed a pathological gambling etiological model based on a modified Kendler's developmental model for major depression, and found it can be used to statistically predict lifetime and past-year pathological gambling through possible risk factors in several developmental levels. Factors statistically predictive of lifetime gambling included family history of substance-use disorders or depression, impulsivity, childhood-onset anxiety, and independent stressful life events. Meanwhile, lifetime history of pathological gambling, personality disorder, and past-year nicotine dependence statistically predicted past-year pathological gambling. Future model development and comparisons between national data sets will be interesting avenues for theoretical advancement.

\section{Sociodemographics and potential risk factors}

Most articles examined elements of sociodemographics and potential risk factors among specified research questions. One of the earliest investigations on gender-related differences by ethnicity found that Black women were more likely than Black men to report pathological gambling, but Hispanic men were more likely than Hispanic women to report subclinical pathological gambling, while no gender differences were observed among White individuals (Blanco et al., 2006). Further analyses by age among individuals with subclinical pathological gambling revealed that more men were in the 18- to 29-year age group, while more women were in the 65 years or more age group. Stronger associations between pathological gambling and psychiatric disorders - mood, anxiety and personality disorders - were evident among women as compared with men (Petry et al., 2005), and among American Indian/Alaskan Native adults (Kong et al., 2016). Problem/pathological gambling prevalence rates were $0.70 \%$ among men and $0.40 \%$ among women (Desai \& Potenza, 2008). The "telescoping" phenomenon, whereby women show a later onset but quicker duration for the development of gambling problems, was suggested in several studies (Pilver et al., 2013a; Vizcaino et al., 2014). Longitudinal analyses of Wave 1 and 2 data revealed that pathological gambling was linked with an increased 3-year incidence of nicotine dependence among females and alcohol dependence among males (Pilver et al., 2013a). In general, men were more likely than women to engage in gambling activities (Wilson et al., 2015).

The sample of lifetime gamblers was racially $76.8 \%$ White, 20.1\% Black, and 14.2\% Hispanic (Strong \& Kahler, 2007). In a sample of individuals who engaged in gambling $(n=11,153)$, Black $(2.2 \%)$ and Native American/Asian $(2.3 \%)$ individuals reported significantly higher percentages of problem/pathological gambling than White individuals (1.2\%; Alegria et al., 2009). Similarly, in this sample, lifetime conditional prevalence of problem/pathological gambling was higher among Black $(9.0 \%)$ and Native Americans/Asian (8.2\%) adults than White (4.0\%) adults. However, in a sample selection of 31,830 white and Hispanic adults, White individuals $(0.5 \%)$ were more likely to exhibit problem/pathological gambling as compared to Hispanic individuals (0.4\%; Barry et al., 2011b). Racial comparisons based on a sample of 32,316 White and Black adults revealed that problem/pathological gambling rates were higher for Black $(0.96 \%)$ than White $(0.45 \%)$ individuals (Barry et al., 2011a). American Indian/Alaskan Native as compared with other Americans (using the total 43,093 Wave 1 sample) were least likely to report 
non-/low-frequency gambling (American Indian/Alaskan Native: $66.5 \%$, White: $70.5 \%$, Black: $72.8 \%$, and other race: $72.3 \%$ ) and most likely to report low-risk gambling (American Indian/Alaskan Native: 30.1\%, White: 26.5\%, Black: 23.4\%, and other race: $24.7 \%$; Kong et al., 2016). It is important to note that percentages vary according to specified sample parameters of each study based on the NESARC data.

Descriptive results were provided in most studies in relation to age, and a few focused on pathological gambling among older adults. Prevalence estimates based on a sample of 25,485 individuals 40 years and above revealed that problem/pathological gambling rates are similar $(0.30 \%)$ for both the younger group (40-64 years) and older group (more than 64 years; Desai et al., 2007). Problem/ pathological gambling in the younger group was linked to poorer subjective health, nicotine dependence, alcohol abuse/dependence, obesity, and one or more chronic conditions, while similar non-significant patterns were seen among the older group. Another study that sampled 10,563 older adults aged 60 or more years found that individuals with problem/pathological gambling as compared to those with recreational gambling (i.e., met 0-2 DSM-IV criteria) had higher rates of alcohol, nicotine, mood, anxiety, personality disorders and obesity, and lower rates of arteriosclerosis or cirrhosis (Pietrzak et al., 2007). Recreational gambling was relatively common among older adults as $30 \%$ reported ever gambling more than 5 times per year, whereas pathological gambling was rare as $0.30 \%$ of older adults met lifetime pathological-gambling and $0.10 \%$ met past-year pathological-gambling diagnostic criteria. Younger gamblers demonstrate loss chasing at lower levels of problem-gambling severity than older gamblers (Strong \& Kahler, 2007).

\section{Psychiatric comorbidities}

Twelve articles provided results on psychiatric comorbidities with pathological gambling, while eight studies investigated comorbidities amid other central variables. Individuals with problem/pathological gambling were more likely to report lifetime psychiatric disorders (Pietrzak et al., 2007), and this pattern was evident across racial/ethnic groups (Barry et al., 2011a, 2011b). Furthermore, past-year problem/pathological gambling was linked to increased odds of the incidence of some Axis I disorders at 3-year follow-up, and these relationships remained significant after adjusting for the effects of covariates (Chou \& Afifi, 2011). Associations between problem-gambling severity and psychiatric disorders are largely not modified by pain interference (Barry et al., 2013). Anxiety disorders accounted for some of the variance in the association between problem-gambling severity and psychopathology, particularly internalizing disorders, tobacco smoking, and multiple personality disorders (Giddens et al., 2012). Anxiety-by-gambling interactions indicate stronger associations between problem-gambling severity and psychiatric disorders among individuals without anxiety disorders than with anxiety disorders, suggesting that some of the variance in the relationships between problem-gambling severity and psychopathology are accounted for by anxiety disorders.
Among respondents with social-anxiety disorder, alcoholuse disorders were linked to substance-use disorders, pathological gambling, and antisocial-personality disorder (Schneier et al., 2010).

Problem/pathological gambling was found to be associated with elevated odds for most Axis I and II disorders among a non-alcohol-use-disorder group, with alcohol-use disorders moderating the relationships between problemgambling severity and psychopathologies (Brewer et al., 2010). Longitudinal analyses at 3 years after initial intake interview indicated that individuals reporting any gambling behavior at baseline as compared to non-gamblers were at increased risk of mood, anxiety, or substance-use disorders (Parhami, Mojtabai, Rosenthal, Afifi, \& Fong, 2014). Income level moderated the relationship between problem/ pathological gambling and alcohol-use disorders, as a middle/higher-income as compared to a lower-income group evidenced stronger associations between problem-gambling severity and alcohol-use disorders (Sanacora, Whiting, Pilver, Hoff, \& Potenza, 2016). Gender-related differences were evident in motivations for gambling and smoking, whereby psychosocial stress and negative mood states were identified to be potential triggers of substance-use disorders and problem/pathological gambling (Pilver et al., 2013a). Impulse-control deficits and increased odds of pathological gambling were linked to a spectrum of antisocial-personality disorder behaviors, such as shoplifting behavior (Blanco et al., 2008), violent behavior (Pulay et al., 2008), cruelty to animals (Vaughn et al., 2009), and fire-setting (Blanco et al., 2010).

Findings from the NESARC on psychiatric comorbidities of pathological gambling have identified common links between pathological gambling and substance-use disorders, alcohol-use disorders, antisocial-personality disorders, and mood disorders. Differences between genders were reported in the etiology and presenting problems associated with problem/pathological-gambling. Future research and clinical practice would benefit from carefully identifying comorbid psychopathology and assessing cluster symptoms of related disorders.

\section{Physical health and chronic medical conditions findings}

Four articles presented findings on physical health and medical conditions in relation to problem/pathological gambling. Higher problem-gambling severity was associated with current obesity status and poorer appraisal of physical health (Morasco et al., 2006). In the same study, at-risk/ problem/pathological gambling was associated with a greater likelihood of utilizing medical services such as the emergency department and being admitted for severe injuries. Another study reported significant associations between recreational gambling and health, with recreational gambling among older adults associated with both negative measures like obesity and positive measures like better physical and mental functioning (Desai et al., 2007).

Prevalence of problem/pathological gambling was higher for individuals with moderate/severe pain interference $(0.79 \%)$ than for those with no/low pain interference (no/low pain interference; 0.48\%; Barry et al., 2013). Prospective evaluations of Wave 1 and 2 NESARC data found 
that the at-risk/problem/pathological-gambling group had a $36 \%$ increased likelihood of developing arteriosclerosis as compared to the non-at-risk/problem/pathological-gambling group (Pilver \& Potenza, 2013). In contrast, the incidence of any liver condition was higher in the non-at-risk/problem/ pathological-gambling group than in the at-risk/problem/ pathological-gambling group; however, this finding did not survive adjustment for covariates.

\section{DISCUSSION}

This systematic review provides a comprehensive synthesis of gambling-related NESARC findings in 51 published articles. Although all studies utilized the same NESARC Wave 1 and/or Wave 2 data, there were variations in terminologies (e.g., pathological gambling, problem/pathological gambling, and at-risk/problem/pathological gambling), thresholds employed, and gambling groups used in describing the problem-gambling-severity continuum - all of which contribute to challenges in comparative analyses between papers. The studies, however, provide important findings related to prevalence estimates of pathological gambling in subgroups and in the general population and information on psychiatric comorbidities, sociodemographic correlates, and gambling typologies. Lifetime rates of pathological gambling $(0.42 \%)$ based on the US NESARC data resembled rates reported in other countries, specifically New Zealand and Sweden. The results from the US NCS-R study also yielded a similar prevalence rate of $0.6 \%$ for pathological gambling in the general population (Kessler et al., 2008). Although rates of pathological gambling appear low, variability is noted across subgroups, and this comprehensive review of NESARC studies sheds light on some health and psychological correlates of pathological gambling and subdiagnostic at-risk/problem gambling found in the US population. Moreover, systematic review of all gambling-related NESARC publications permits comparative evaluation, reflection, and formulation of strategic areas for future directions, specifically public health campaigns aimed at reducing the occurrence of problem gambling among vulnerable groups.

A key strength of the NESARC studies is the utilization of the psychometrically valid and reliable AUDADIS-IV. Good reliability indicators for alcohol consumption and psychiatric disorders suggest that it is a useful measurement tool in varied research contexts, especially in population studies - the target sample for which it was developed (Grant, Dawson, et al., 2003). With the passage of the DSM5, pathological gambling was renamed gambling disorder and moved from an impulse-control disorder to addictive disorder and subsequently grouped with substance-use disorders. The diagnostic criteria of gambling disorder remained similar except for the elimination of the illegalacts criterion and the lowering of the threshold from five to four diagnostic criteria (Petry, Blanco, Jin, \& Grant, 2014). Because gambling disorder occurs at a low base rate in the general population and as one means of improving statistical power in some studies, problem gambling and pathological gambling have often been combined into one category problem/pathological gambling. Careful formulation of subsequent versions of the AUDADIS provides an additional benefit of allowing prospective longitudinal and comparative analyses between Wave 1 and Wave 2 data for most psychiatric disorders, with an exception of pathological gambling. Although there were other additional assessments in Wave 2 as compared with Wave 1, pathological gambling measurements were omitted from Wave 2 data collection, which limits the possibility of performing longitudinal prospective analyses on gambling constructs using NESARC data, particularly with respect to incident pathological gambling or documenting changes in rates of problem-gambling severity among specific groups over time. Longitudinal data examining both risk and protective factors associated with gambling disorder are needed, particularly given the expansion of gambling, particularly sports gambling but also casino and other forms, occurring in the USA. Given the wealth of knowledge gained from the NESARC specific to gambling disorder, a new longitudinal prospective study is needed to tackle many unanswered questions around the prevalence, severity, and course of gambling disorder in the USA.

The results reported from the NESARC on potential risk factors and sociodemographic correlates of pathological gambling resonate with prior findings. Elevated odds of pathological gambling was associated with being male, Black, aged between 45 and 64 years and widowed/separated/divorced. Gender-related differences varied when the sample was stratified into racial/ethnic groups, whereby higher prevalence of pathological gambling was reported among Hispanic men and Black women, while no differences were observed among White men/women (Blanco et al., 2006). Positive associations were evident between pathological gambling and other psychopathology - substance dependence, mood disorders, personality disorders (particularly antisocial-personality disorder), comorbid diagnoses (stronger among females), and suicidal attempts (Moghaddam et al., 2015). Furthermore, higher income increases the positive association between problem-gambling severity and alcohol dependence. Wave 1 problem/pathological gambling prospectively increased likelihood of predicting Wave 2 incidence of mood disorders, generalized anxiety disorder, posttraumatic stress disorder, and alcohol abuse/dependence (Chou \& Afifi, 2011).

Recreational gambling among older adults ( $>65$ years) was linked with better self-reported physical and mental health, despite increased odds of negative health measures such as obesity (Desai et al., 2007). Older adults with presenting at-risk/problem and pathological gambling should be closely observed for physical health conditions. Treatment-seeking rates among individuals with pathological gambling were low in the entire population with no differences reported between those with early- and later-onset pathological gambling. These findings were generally consistent with national data sets worldwide with specific variations (intricate to US demographics) in prospective analyses, ethnicity differences, and recreational gambling among older adults.

The US NESARC data structure has provided some avenues for testing theoretical models and analyzing symptom patterns, which were main aims of selected studies reported here. While investigations on prevalence, descriptive estimates, and behavioral correlates are important outputs from national data sets, it is beneficial to examine empirically 
and test theoretically driven models of pathologicalgambling etiology using these nationally representative data. Analyses of NESARC data validated the use of a total pathological-gambling score to consider problemgambling severity and revealed group differences in symptom endorsements (see Sacco et al., 2011). Careful adaptation of observed data on latent structures of proposed models such as the pathways model (Nower et al., 2013; Valleur et al., 2015) and cognitive-behavioral model of gambling behavior (Raylu, Oei, Loo, \& Tsai, 2016) provide further evidence that may help generate future evolutions of theoretical frameworks, which further guide treatment formulations and prevention initiatives for vulnerable subgroups. The results on potential risk factors for early- versus later-onset pathological gambling (Vizcaino et al., 2014) and etiological or ecological determinants of gambling subtypes (Blanco et al., 2015; Carragher \& McWilliams, 2011) may enable future identification of atrisk groups for early intervention, inform assessment of possible behavioral addictions at intake interviews, and highlight key areas of focus in population studies. Scientific prediction of future behavior based on potential risk factors should be used with caution to reduce occurrences of false positives or negatives in case assessments. Future research, not covered in NESARC, is needed to identify protective factors against the development of problem/pathological gambling. Since the NESARC study was initially conducted, additional research has been focused on identifying protective factors for youth and other vulnerable groups to reduce early onset of gambling disorder (Dowling et al., 2017), yet our understanding of specific protective factors for vulnerable groups (e.g., youth, ethnic minorities, and military veterans) remains limited. In these efforts, not only should DSM-5 criteria for gambling disorder be considered, but also the criteria for gambling disorder and hazardous gambling or betting in the 11th edition of the International Classification of Diseases, with the latter perhaps particularly well suited for promoting public health through identification and targeting of potentially vulnerable groups (World Health Organization, 2018).

The 51 NESARC studies published on problem/ pathological gambling provide a wide array of empirical findings that may help guide future research. It would have been ideal if Wave 2 had included gambling-related measures. This would have allowed for longitudinal analyses of development and/or recovery, while answering questions in relation to telescoping effects, natural recovery, and pathway and other models of transitions. Although eight studies in this review provided some prospective analyses using both Wave 1 and 2 data sets, there were no comparative analyses on gambling-related outcomes, given the absence of gambling-related measures in Wave 2. In addition, oversampling of individuals of Black or Hispanic backgrounds and young adults at the design-phase of the survey may have provided an overrepresentation of these cohorts in outcome analyses. In studies employing the NESARC-generated weights, analyses could statistically adjust for oversampling to be representative of the US population, which presents itself as an important alternative to stratified random sampling of US households given important questions that could be addressed as the result of oversampling.
In conclusion, the first review of gambling-related NESARC findings revealed that pathological gambling rarely exists in isolation and is commonly associated with substance-use, mood, anxiety, and personality disorders. This confirms, in a nationally representative data set, past findings from other cross-sectional investigations. Gender, racial/ ethnic, and sociodemographic variability were identified across comorbidities, which may facilitate early identification of at-risk groups for prevention efforts. As rates of adverse childhood experiences and suicidal attempts were higher among individuals with problem and pathological gambling, further investigations and assessments are important to clarify the underlying mechanisms of these associations. It is noteworthy that first-generation immigrants show better health outcomes and lower pathological-gambling prevalence as compared to second- and third-generation immigrants in the US population (i.e., the immigrant paradox), which is in contrast to past research that highlighted higher rates of pathological gambling among migrant populations (Petry, Armentano, Kuoch, Norinth, \& Smith, 2003). The finding highlights the importance of stratifying generational immigrant status in measuring prevalence or outcome indicators. Although recreational gambling among older adults is relatively common, lifetime and past-year diagnoses of pathological gambling were rare. Future research among older adults and treatment-seeking individuals are potential avenues for further investigation stemming from NESARC findings.

Funding sources: This study was supported by the Monash University's Outside Studies Program and a Center of Excellence grant from the National Center for Responsible Gaming. No funding agencies had input into the content of this manuscript, and none of the authors have any relevant conflicts of interests with the content of this manuscript.

Authors' contribution: JMYL and MNP designed the study and wrote the protocol. JMYL conducted the literature search, extracted the data, and wrote the first draft of the manuscript. SWK and MNP reviewed the literature search, data extraction, and contributed to the manuscript. All authors contributed to and have approved the final version of the manuscript.

Conflict of interest: MNP has consulted for Rivermend Health, Opiant Therapeutics, Game Day Data, and the Addiction Policy Forum; has received research support from Mohegan Sun Casino and the National Center for Responsible Gaming; has participated in surveys, mailings, or telephone consultations related to drug addiction, impulsecontrol disorders or other health topics; has consulted for gambling and legal entities on issues related to impulsecontrol/addictive disorders; provides clinical care in a problem gambling services program; has performed grant reviews for the National Institutes of Health and other agencies; has edited journals and journal sections; has given academic lectures in grand rounds, CME events and other clinical or scientific venues; and has generated books or book chapters for publishers of mental health texts. The other authors have no conflict of interest. 


\section{REFERENCES}

Alegria, A. A., Petry, N. M., Hasin, D. S., Liu, S. M., Grant, B. F., \& Blanco, C. (2009). Disordered gambling among racial and ethnic groups in the US: Results from the National Epidemiologic Survey on Alcohol and Related Conditions. CNS spectrums, 14(3), 132-142. doi:10.1017/s1092852900020113

American Psychiatric Association. (2000). Diagnostic and statistical manual of mental disorders: DSM-IV-TR (4th ed., text rev.). Washington, DC: American Psychiatric Association.

American Psychiatric Association. (2013). Diagnostic and statistical manual of mental disorders: DSM-5 (5th ed.). Washington, DC: American Psychiatric Association.

Barry, D. T., Pilver, C. E., Hoff, R. A., \& Potenza, M. N. (2013). Pain interference, gambling problem severity, and psychiatric disorders among a nationally representative sample of adults. Journal of Behavioral Addictions, 2(3), 138-144. doi:10.1556/ JBA.2.2013.010

Barry, D. T., Stefanovics, E. A., Desai, R. A., \& Potenza, M. N. (2011a). Differences in the associations between gambling problem severity and psychiatric disorders among Black and White adults: Findings from the National Epidemiologic Survey on Alcohol and Related Conditions. American Journal on Addictions, 20(1), 69-77. doi:10.1111/j.1521-0391.2010. 00098.x

Barry, D. T., Stefanovics, E. A., Desai, R. A., \& Potenza, M. N. (2011b). Gambling problem severity and psychiatric disorders among Hispanic and White adults: Findings from a nationally representative sample. Journal of Psychiatric Research, 45(3), 404-411. doi:10.1016/j.jpsychires.2010.07.010

Bernardi, S., Petry, N. M., Martins, S. S., Hasin, D. S., Liu, S., Grant, B. F., \& Blanco, C. (2019). Predictors of remission from problem and pathological gambling: Results from the National Epidemiologic Survey on Alcohol and Related Conditions (NESARC). Archives of Behavioral Addictions, 1(1). doi:10.30435/ ABA.01.2019.04

Binde, P. (2014). Gambling in Sweden: The cultural and sociopolitical context. Addiction, 109(2), 193-198. doi:10.1111/ add. 12103

Blanco, C., Alegria, A. A., Petry, N. M., Grant, J. E., Simpson, H. B., Liu, S. M., Grant, B. F., \& Hasin, D. S. (2010). Prevalence and correlates of fire-setting in the United States: Results from the National Epidemiologic Survey on Alcohol and Related Conditions (NESARC). Journal of Clinical Psychiatry, 71(9), 1218-1225. doi:10.4088/ JCP.08m04812gry

Blanco, C., Grant, J., Petry, N. M., Simpson, H. B., Alegria, A., Liu, S. M., \& Hasin, D. (2008). Prevalence and correlates of shoplifting in the United States: Results from the National Epidemiologic Survey on Alcohol and Related Conditions (NESARC). American Journal of Psychiatry, 165(7), 905-913. doi:10.1176/appi. ajp.2008.07101660

Blanco, C., Hanania, J., Petry, N. M., Wall, M. M., Wang, S., Jin, C. J., \& Kendler, K. S. (2015). Towards a comprehensive developmental model of pathological gambling. Addiction, 110(8), 1340-1351. doi:10.1111/add.12946

Blanco, C., Hasin, D. S., Petry, N., Stinson, F. S., \& Grant, B. F. (2006). Sex differences in subclinical and DSM-IV pathological gambling: Results from the National Epidemiologic Survey on Alcohol and Related Conditions. Psychological Medicine, 36(7), 943-953. doi:10.1017/S0033291706007410
Boudreau, A., Labrie, R., \& Shaffer, H. J. (2009). Towards DSMV: 'Shadow Syndrome' symptom patterns among pathological gamblers. Addiction Research \& Theory, 17(4), 406-419. doi:10.1080/16066350902802301

Brewer, J. A., Potenza, M. N., \& Desai, R. A. (2010). Differential associations between problem and pathological gambling and psychiatric disorders in individuals with and without alcohol abuse or dependence. CNS Spectrums, 15(1), 33-44. doi:10.1017/S1092852900000286

Carragher, N., \& McWilliams, L. A. (2011). A latent class analysis of DSM-IV criteria for pathological gambling: Results from the National Epidemiologic Survey on Alcohol and Related Conditions. Psychiatry Research, 187(1-2), 185-192. doi:10.1016/ j.psychres.2010.12.022

Chou, K. L., \& Afifi, T. O. (2011). Disordered (pathologic or problem) gambling and axis I psychiatric disorders: Results from the National Epidemiologic Survey on Alcohol and Related Conditions. American Journal of Epidemiology, 173(11), 1289-1297. doi:10.1093/aje/kwr017

Chou, K. L., \& Cheung, K. C. (2013). Major depressive disorder in vulnerable groups of older adults, their course and treatment, and psychiatric comorbidity. Depression and Anxiety, 30(6), 528-537. doi:10.1002/da.22073

Cowlishaw, S., \& Hakes, J. K. (2015). Pathological and problem gambling in substance use treatment: Results from the National Epidemiologic Survey on Alcohol and Related Conditions (NESARC). American Journal on Addictions, 24(5), 467-474. doi:10.1111/ajad.12242

Cowlishaw, S., Hakes, J. K., \& Dowling, N. A. (2016). Gambling problems in treatment for affective disorders: Results from the National Epidemiologic Survey on Alcohol and Related Conditions (NESARC). Journal of Affective Disorders, 202, 110-114. doi:10.1016/j.jad.2016.05.023

Desai, R. A., Desai, M. M., \& Potenza, M. N. (2007). Gambling, health, and age: Data from the National Epidemiologic Survey on Alcohol and Related Conditions. Psychology of Addictive Behaviors, 21(4), 431-440. doi:10.1037/0893-164X.21.4.431

Desai, R. A., \& Potenza, M. N. (2008). Gender differences in the associations between past-year gambling problems and psychiatric disorders. Social Psychiatry and Psychiatric Epidemiology, 43(3), 173-183. doi:10.1007/s00127-007-0283-Z

Devlin, M. E., \& Walton, D. (2012). The prevalence of problem gambling in New Zealand as measured by the PGSI: Adjusting prevalence estimates using meta-analysis. International Gambling Studies, 12(2), 177-197. doi:10.1080/14459795.2011. 653384

Dowling, N. A., Merkouris, S. S., Greenwood, C. J., Oldenhof, E., Toumbourou, J. W., \& Youssef, G. J. (2017). Early risk and protective factors for problem gambling: A systematic review and meta-analysis of longitudinal studies. Clinical Psychology Review, 51, 109-124. doi:10.1016/j.cpr.2016.10.008

Ekholm, O., Eiberg, S., Davidsen, M., Holst, M., Larsen, C. V., \& Juel, K. (2014). The prevalence of problem gambling in Denmark in 2005 and 2010: A sociodemographic and socioeconomic characterization. Journal of Gambling Studies, 30(1), 1-10. doi:10.1007/s10899-012-9347-4

Fong, D. K., \& Ozorio, B. (2005). Gambling participation and prevalence estimates of pathological gambling in a far-east city: Macao. UNLV Gaming Research \& Review Journal, 9(2), 15-28. Retrieved from https://digitalscholarship.unlv.edu/grrj/ vol $9 /$ iss $2 / 2$ 
Gebauer, L., LaBrie, R., \& Shaffer, H. J. (2010). Optimizing DSMIV-TR classification accuracy: A brief biosocial screen for detecting current gambling disorders among gamblers in the general household population. Canadian Journal of PsychiatryRevue Canadienne De Psychiatrie, 55(2), 82-90. doi:10.1177/ 070674371005500204

Giddens, J. L., Stefanovics, E., Pilver, C. E., Desai, R., \& Potenza, M. N. (2012). Pathological gambling severity and co-occurring psychiatric disorders in individuals with and without anxiety disorders in a nationally representative sample. Psychiatry Research, 199(1), 58-64. doi:10.1016/ j.psychres.2012.03.052

Grant, B. F., \& Dawson, D. A. (2006). Introduction to the National Epidemiologic Survey on Alcohol and Related Conditions. Alcohol Health \& Research World, 29(2), 74-78. Retrieved from https://www.ncbi.nlm.nih.gov/pmc/articles/PMC6527251/

Grant, B. F., Dawson, D. A., Stinson, F. S., Chou, P. S., Kay, W., \& Pickering, R. (2003). The Alcohol Use Disorder and Associated Disabilities Interview Schedule-IV (AUDADIS-IV): Reliability of alcohol consumption, tobacco use, family history of depression and psychiatric diagnostic modules in a general population sample. Drug and Alcohol Dependence, 71(1), 7-16. doi:10.1016/S0376-8716(03)00070-X

Grant, B. F., Moore, T., Shepard, J., \& Kaplan, K. (2003). Source and accuracy statement: Wave 1 National Epidemiologic Survey on alcohol and Related Conditions (NESARC). Bethesda, MD: National Institute on Alcohol Abuse and Alcoholism.

Grant, J. E., Desai, R. A., \& Potenza, M. N. (2009). Relationship of nicotine dependence, subsyndromal and pathological gambling, and other psychiatric disorders: Data from the National Epidemiologic Survey on Alcohol and Related Conditions. Journal of Clinical Psychiatry, 70(3), 334-343. doi:10.4088/ JCP. $08 \mathrm{~m} 04211$

Hammond, C. J., Shirk, S. D., Foster, D. W., Potenza, N. B., Kraus, S. W., Mayes, L. C., Hoff, R. A., \& Potenza, M. N. (2019). Cannabis use, problem-gambling severity, and psychiatric disorders: Data from the National Epidemiological Survey on Alcohol and Related Conditions. Psychology of Addictive Behaviors. Advance online publication. doi:10.1037/adb0000472

Kessler, R. C., Berglund, P. A., Chiu, W. T., Demler, O., Glantz, M., Lane, M. C., Jin, R., Merikangas, K. R., Nock, M., Olfson, M., Pincus, H. A., Walters, E. E., Wang, P. S., \& Wells, K. B. (2008). The National Comorbidity Survey Replication (NCS$\mathrm{R})$ : Cornerstone in improving mental health and mental health care in the United States. In R. C. Kessler \& T. B. Üstün (Eds.), The WHO Mental Health Surveys: Global perspectives on the epidemiology of mental disorders (pp. 165-209). New York: Cambridge University.

Kong, G., Smith, P. H., Pilver, C., Hoff, R., \& Potenza, M. N. (2016). Problem-gambling severity and psychiatric disorders among American-Indian/Alaska native adults. Journal of Psychiatric Research, 74, 55-62. doi:10.1016/j.jpsychires.2015.12.004

Krueger, R. F. (1999). The structure of common mental disorders. Archives of General Psychiatry, 56(10), 921-926. doi:10.1001/ archpsyc.56.10.921

Loo, J. M. Y., \& Ang, K. T. (2013). Prevalence of problem gambling in Selangor urban areas. Kuala Lumpur: Malaysia: Monash University Malaysia \& Malaysian Mental Health Association.

Loo, J. M. Y., Raylu, N., \& Oei, T. P. S. (2008). Gambling among the Chinese: A comprehensive review. Clinical Psychology Review, 28(7), 1152-1166. doi:10.1016/j.cpr.2008.04.001
Moghaddam, J. F., Dickerson, D. L., Yoon, G., \& Westermeyer, J. (2014). Nicotine dependence and psychiatric and substance use disorder comorbidities among American Indians/Alaska Natives: Findings from the National Epidemiologic Survey on Alcohol and Related Conditions. Drug and Alcohol Dependence, 144, 127-133. doi:10.1016/j.drugalcdep.2014.08.017

Moghaddam, J. F., Yoon, G., Dickerson, D. L., Kim, S. W., \& Westermeyer, J. (2015). Suicidal ideation and suicide attempts in five groups with different severities of gambling: Findings from the National Epidemiologic Survey on Alcohol and Related Conditions. American Journal on Addictions, 24(4), 292-298. doi:10.1111/ajad.12197

Moher, D., Liberati, A., Tetzlaff, J., Altman, D. G., \& PRISMA Group. (2009). Preferred Reporting Items for Systematic Reviews and Meta-Analyses: The PRISMA statement. Annals of Internal Medicine, 151(4), 264-269. doi:10.7326/00034819-151-4-200908180-00135

Morasco, B. J., Pietrzak, R. H., Blanco, C., Grant, B. F., Hasin, D., $\&$ Petry, N. M. (2006). Health problems and medical utilization associated with gambling disorders: Results from the National Epidemiologic Survey on Alcohol and Related Conditions. Psychosomatic Medicine, 68(6), 976-984. doi:10.1097/01.psy. 0000238466.76172.cd

National Council on Problem Gambling. (2014). Report of survey on participation in gambling activities among Singapore residents. Retrieved from http://app.msf.gov.sg/Research-Room/ Research-Statistics/Survey-on-Gambling-Participation-AmongSpore

Nelson, S. E., Gebauer, L., LaBrie, R. A., \& Shaffer, H. J. (2009). Gambling problem symptom patterns and stability across individual and timeframe. Psychology of Addictive Behaviors, 23(3), 523-533. doi:10.1037/a0016053

Nicholson, R., Mackenzie, C., Afifi, T. O., Keough, M., \& Sareen, J. (2019). An examination of comorbid psychiatric disorders in disordered gamblers versus other substance-related disorders. Journal of Gambling Studies, 35(3), 829-847. doi:10.1007/ s10899-019-09839-y

Nower, L., Martins, S. S., Lin, K. H., \& Blanco, C. (2013). Subtypes of disordered gamblers: Results from the National Epidemiologic Survey on Alcohol and Related Conditions. Addiction, 108(4), 789-798. doi:10.1111/add.12012

Oleski, J., Cox, B. J., Clara, I., \& Hills, A. (2011). Pathological gambling and the structure of common mental disorders. Journal of Nervous and Mental Disease, 199(12), 956-960. doi:10.1097/NMD.0b013e3182392931

Parhami, I., Mojtabai, R., Rosenthal, R. J., Afifi, T. O., \& Fong, T. W. (2014). Gambling and the onset of comorbid mental disorders: A longitudinal study evaluating severity and specific symptoms. Journal of Psychiatric Practice, 20(3), 207-219. doi:10.1097/01.pra.0000450320.98988.7c

Petry, N. M., Armentano, C., Kuoch, T., Norinth, T., \& Smith, L. (2003). Gambling participation and problems among South East Asian refugees to the United States. Psychiatric Services, 54(8), 1142-1148. doi:10.1176/appi.ps.54.8.1142

Petry, N. M., Blanco, C., Jin, C., \& Grant, B. F. (2014). Concordance between gambling disorder diagnoses in the DSM-IV and DSM-5: Results from the National Epidemiological Survey of Alcohol and Related Disorders. Psychology of Addictive Behaviors, 28(2), 586-591. doi:10.1037/a0034661

Petry, N. M., Stinson, F. S., \& Grant, B. F. (2005). Comorbidity of DSM-IV pathological gambling and other psychiatric 
disorders: Results from the National Epidemiologic Survey on Alcohol and Related Conditions. Journal of Clinical Psychiatry, 66(5), 564-574. doi:10.4088/JCP.v66n0504

Pietrzak, R. H., Morasco, B. J., Blanco, C., Grant, B. F., \& Petry, N. M. (2007). Gambling level and psychiatric and medical disorders in older adults: Results from the National Epidemiologic Survey on Alcohol and Related Conditions. American Journal of Geriatric Psychiatry, 15(4), 301-313. doi:10.1097/ 01.JGP.0000239353.40880.cc

Pilver, C. E., Libby, D. J., Hoff, R. A., \& Potenza, M. N. (2013a). Gender differences in the relationship between gambling problems and the incidence of substance-use disorders in a nationally representative population sample. Drug and Alcohol Dependence, 133(1), 204-211. doi:10.1016/j.drugalcdep.2013.05.002

Pilver, C. E., Libby, D. J., Hoff, R. A., \& Potenza, M. N. (2013b). Problem gambling severity and the incidence of Axis I psychopathology among older adults in the general population. Journal of Psychiatric Research, 47(4), 534-541. doi:10.1016/ j.jpsychires.2012.12.013

Pilver, C. E., \& Potenza, M. N. (2013). Increased incidence of cardiovascular conditions among older adults with pathological gambling features in a prospective study. Journal of Addiction Medicine, 7(6), 387-393. doi:10.1097/ADM.0b013e31829e9b36

Productivity Commission. (2010). Gambling: Productivity commission inquiry report no. 50. Canberra, Australia: Productivity Commission. Retrieved from https://www.pc.gov.au/inquiries/ completed/gambling-2010/report/gambling-report-volume1.pdf

Pulay, A. J., Dawson, D. A., Hasin, D. S., Goldstein, R. B., Ruan, J., Pickering, R. P., Huang, B., Chou, S. P., \& Grant, B. F. (2008). Violent behavior and DSM-IV psychiatric disorders: Results from the National Epidemiologic Survey on alcohol and related conditions. Journal of Clinical Psychiatry, 69(1), 12-22. doi:10.4088/JCP.v69n0103

Raylu, N., Oei, T. P. S., Loo, J. M. Y., \& Tsai, J.-S. (2016). Testing the validity of a cognitive behavioral model for gambling behavior. Journal of Gambling Studies, 32(2), 773-788. doi:10.1007/s10899-015-9567-5

Roberts, A., Landon, J., Sharman, S., Hakes, J., Suomi, A., \& Cowlishaw, S. (2018). Gambling and physical intimate partner violence: Results from the National Epidemiologic Survey on Alcohol and Related Conditions (NESARC). The American Journal on Addictions, 27(1), 7-14. doi:10.1111/ajad.12656

Ronzitti, S., Kraus, S. W., Hoff, R. A., Clerici, M., \& Potenza, M. N. (2018). Problem-gambling severity, suicidality and DSM-IV Axis II personality disorders. Addictive Behaviors, 82, 142-150. doi:10.1016/j.addbeh.2018.03.003

Ronzitti, S., Kraus, S. W., Hoff, R. A., Clerici, M., \& Potenza, M. N. (2019). Problem-gambling severity, suicidality, and DSM-IV Axis I psychiatric disorders. International Journal of Mental Health and Addiction. Advance online publication. 1-16. doi:10.1007/s11469-019-00080-7

Sacco, P., Torres, L. R., Cunningham-Williams, R. M., Woods, C., \& Unick, G. (2011). Differential item functioning of pathological gambling criteria: An examination of gender, race/ ethnicity, and age. Journal of Gambling Studies, 27(2), 317-330. doi:10.1007/s10899-010-9209-x

Sanacora, R. L., Whiting, S. W., Pilver, C. E., Hoff, R. A., \& Potenza, M. N. (2016). Relationships between problemgambling severity and psychopathology as moderated by income. Journal of Behavioral Addictions, 5(3), 429-438. doi:10.1556/2006.5.2016.045
Schneier, F. R., Foose, T. E., Hasin, D. S., Heimberg, R. G., Liu, S. M., Grant, B. F., \& Blanco, C. (2010). Social anxiety disorder and alcohol use disorder co-morbidity in the National Epidemiologic Survey on Alcohol and Related Conditions. Psychological Medicine, 40(6), 977-988. doi:10.1017/S0033291709991231

Sharma, A., \& Sacco, P. (2015). Adverse childhood experiences and gambling: Results from a national survey. Journal of Social Work Practice in the Addictions, 15(1), 25-43. doi:10.1080/1533256X.2015.996502

Slutske, W. S. (2006). Natural recovery and treatment-seeking in pathological gambling: Results of two U.S. national surveys. American Journal of Psychiatry, 163(2), 297-302. doi:10.1176/ appi.ajp.163.2.297

Strong, D. R., \& Kahler, C. W. (2007). Evaluation of the continuum of gambling problems using the DSM-IV. Addiction, 102(5), 713-721. doi:10.1111/j.1360-0443.2007.01789.x

Valleur, M., Codina, I., Venisse, J. L., Romo, L., Magalon, D., Fatseas, M., Chéreau-Boudet, I., Gorsane, M. A., Guilleux, A., Groupe, J. E. U., Grall-Bronnec, M., \& Challet-Bouju, G. (2015). Towards a validation of the three pathways model of pathological gambling. Journal of Gambling Behavior, 32(2), 757-7711. doi:10.1007/s10899-015-9545-y

Vaughn, M. G., Fu, Q., DeLisi, M., Beaver, K. M., Perron, B. E., Terrell, K., \& Howard, M. O. (2009). Correlates of cruelty to animals in the United States: Results from the National Epidemiologic Survey on Alcohol and Related Conditions. Journal of Psychiatric Research, 43(15), 1213-1218. doi:10.1016/ j.jpsychires.2009.04.011

Vizcaino, E. J. V., Fernandez-Navarro, P., Petry, N., Rubio, G., \& Blanco, C. (2014). Differences between early-onset pathological gambling and later-onset pathological gambling: Data from the National Epidemiologic Survey on Alcohol and Related Conditions (NESARC). Addiction, 109(5), 807-813. doi:10.1111/ add. 12461

Wardle, H., Moody, A., Spence, S., Orford, J., Volberg, R., Jotangia, D., Griffiths, M., Hussey, D., \& Dobbie, F. (2011). British gambling prevalence survey 2010. Retrieved from http://www.gamblingcommission.gov.uk/

Wilson, A. N., Salas-Wright, C. P., Vaughn, M. G., \& Maynard, B. R. (2015). Gambling prevalence rates among immigrants: A multigenerational examination. Addictive Behaviors, 42, 79-85. doi:10.1016/j.addbeh.2014.11.003

Wong, I. L., \& So, E. M. (2003). Prevalence estimates of problem and pathological gambling in Hong Kong. American Journal of Psychiatry, 160(7), 1353-1354. doi:10.1176/appi.ajp.160.7.1353

World Health Organization. (1992). The ICD-10 classification of mental and behavioural disorders: Clinical descriptions and diagnostic guidelines. Geneva, Switzerland: WHO.

World Health Organization. (2018). International classification of diseases for mortality and morbidity statistics (11th Rev.). Retrieved from https://icd.who.int/browse11/1-m/en

Wu, A. M., Lai, M. H., \& Tong, K. K. (2014). Gambling disorder: Estimated prevalence rates and risk factors in Macao. Psychology of Addictive Behaviors, 28(4), 1190-1197. doi:10.1037/ a0037603

Wu, L. T., Woody, G. E., Yang, C. M., \& Blazer, D. G. (2011). How do prescription opioid users differ from users of heroin or other drugs in psychopathology: Results from the National Epidemiologic Survey on Alcohol and Related Conditions. Journal of Addiction Medicine, 5(1), 28-35. doi:10.1097/ ADM.0b013e3181e0364e 